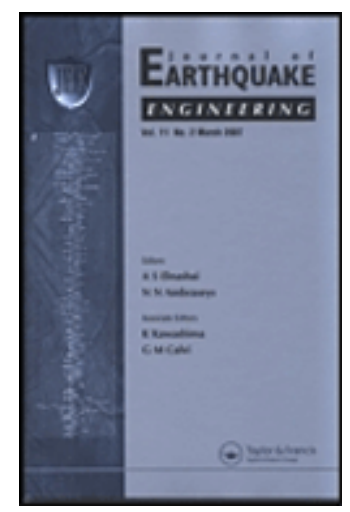

\title{
Analytical derivation and experimental verification of overstrength factors of dowel-type timber connections for capacity design
}

\begin{tabular}{|r|l|}
\hline Journal: & Journal of Earthquake Engineering \\
\hline Manuscript ID & UEQE-2019-4029.R2 \\
\hline Manuscript Type: & Full Length Papers \\
\hline Author: & 26-May-2020 \\
\hline Complete List of Authors: & $\begin{array}{l}\text { Ottenhaus, Lisa-Mareike; University of Queensland - Saint Lucia Campus, } \\
\text { School of Civil Engineering } \\
\text { Li, Minghao; University of Canterbury, Civil and Natural Resources } \\
\text { Engineering } \\
\text { Smith, Tobias; PTL I Structural Timber Consultants }\end{array}$ \\
\hline Keywords: & Overstrength, Capacity design, Timber, Connection, Seismic \\
\hline
\end{tabular}


Analytical derivation and experimental verification of overstrength factors of dowel-type timber connections for

\title{
capacity design
}

\author{
Lisa-Mareike Ottenhaus ${ }^{1 *}$, Minghao $\mathrm{Li}^{2}$, Tobias $\mathrm{Smith}^{3}$ \\ ${ }^{1}$ Lecturer, School of Civil Engineering, the University of Queensland, Australia, \\ l.ottenhaus@uq.edu.au; (corresponding author,formerly Ph.D. candidate at University \\ of Canterbury, New Zealand) \\ ${ }^{2}$ Senior Lecturer, Department of Civil and Natural Resources Engineering, University \\ of Canterbury, New Zealand, minghao.li@canterbury.ac.nz; \\ ${ }^{3}$ PTL | Structural Timber Consultants, PO Box 8415, Christchurch 8041, New Zealand. \\ t.smith@ptlnz.com;
}




\title{
Analytical derivation and experimental verification of overstrength factors of dowel-type timber connections for capacity design
}

\begin{abstract}
In timber structures, connections traditionally provide ductility and energy dissipation under seismic loading. Capacity design ensures that the global structural response is ductile by applying overstrength to the design demand of brittle elements. Overstrength is often derived experimentally which is costly and time consuming. This paper proposes an analytical method to estimate the overstrength of dowel-type timber connections based on inherent material properties, thereby reducing the need for experimental testing. The method is validated with data from previous experiments and literature for dowelled, bolted, and nailed timber connections, and provides reasonably accurate upper-bound overstrength estimates.
\end{abstract}

Keywords: overstrength; capacity design; timber; connection; seismic

\section{Introduction}

Capacity design was originally developed in California in the 1960s and was promoted in New Zealand by Tom Paulay for seismic design of reinforced concrete structures (Park and Paulay 1975, Fardis 2018). Nowadays, capacity design is widely accepted in seismic design of steel and concrete structures and has recently gained popularity in the design of timber structures, with suggestions to include it in Chapter 8 of Eurocode 8 for the seismic design of timber structures (Follesa et al. 2011, Loss et al. 2013, Follesa et al. 2018).

Capacity design of timber structures ensures that ductile behaviour can be achieved under seismic loading by detailing ductile connections as the weakest link along the load path, and protecting all brittle timber elements from the overstrength of the ductile connections (Moroder et al. 2014, Fardis 2018). In this context, overstrength of the 
ductile connections is defined as the difference between the design strength, $F_{d}$, and the $95^{\text {th }}$-percentile of the actual strength distribution, $F_{0.95}$ (Jorissen and Fragiacomo 2011, Follesa et al. 2018). To ensure a structural system can mobilise its ductile potential, $F_{B R, d} \geq \gamma_{R d} F_{u, d}$ needs to be satisfied, i.e. the design capacity of the brittle elements, $F_{B R, d}$, needs to be higher than the ultimate actual capacity of the ductile connection, $F_{u, d}$. This realisation leads to the definition of an overstrength factor, $\gamma_{R d}$, that defines the gap between the design and ultimate probable capacity of the ductile connection $\left(F_{0.95}\right)$, beyond which the brittle failure modes should be verified.

Overstrength in timber connections stems from a variety of sources and can be difficult to define accurately. It is often derived experimentally and only limited overstrength data of few timber connection types is currently available in literature.

A component based overstrength concept, adapted from Jorissen and Fragiacomo (2011), is shown for dowel-type timber connections in Figure 1 and Equation 1 and the method presented in this paper is based on this overstrength definition

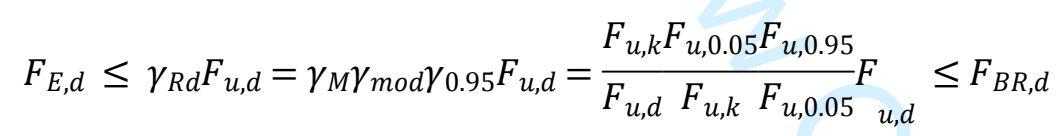

where $F_{E, d}$ is the design demand of the connection, $F_{u}$ is the ultimate ductile connection strength as defined in design codes with its characteristic and design values, $F_{u, k}$ and $F_{u, d}, \gamma_{M}$ is the partial material safety factor, $\gamma_{m o d}$ accounts for conservatism of analytical models for design, and $\gamma_{0.95}$ quantifies the difference between the $5^{\text {th }}$ and $95^{\text {th }}$-percentile of the strength distribution ( $F_{0.05}$, and $F_{0.95}$, respectively).

Since $\gamma_{M}$ is defined in design codes, the only unknown components of the theoretical overstrength, defined as $\gamma_{R d, t h}$, are $\gamma_{\bmod }$ and $\gamma_{0.95}$. A method to estimate these two unknowns will be presented in this paper. While the connection design is based on the 
European yield model (EYM) in Eurocode 5 (2004), the overstrength concept holds for different material standards and material grades alike and reference to the respective material standards is made where appropriate.

It should be noted that there are more refined versions of this approach, such as the modified capacity design model by Trutalli et al. (2018). However, for the purpose of providing an analytical calculation method aimed at designers and practitioners, the method by Jorissen and Fragiacomo (2011) produces reasonably accurate results without introducing too much complexity, as will be presented in this paper.

\section{Overstrength theory in capacity design}

Experimental overstrength factors can be defined by $\gamma_{R d, \text { exp }}=F_{\max , \exp } / F_{u, k, p r e d}$ as shown in Figure 2, where $F_{\max , \exp }$ is the maximum capacity observed across a number of test specimens, and $F_{u, k, p r e d}$ is the predicted ultimate ductile capacity based on the EYM. This method has the drawback that the observed maximum capacity is generally smaller than the $95^{\text {th }}$-percentile of the connection strength distribution $\left(F_{\max , \exp } \leq F_{u, 0.95}\right)$ due to a limited sample size; thus non-conservative overstrength factors may sometimes be derived. Therefore, several researchers attempted to identify individual overstrength components and derived $\gamma_{R d, t h}$ for connections (Jorissen and Fragiacomo 2011, Schick at al. 2013, Brühl et al. 2014, Gavric et al. 2014, Vogt et al. 2014, Izzi et al. 2016, O'Ceallaigh and Harte 2019). An overview of some connection overstrength values in literature for connections in Glued Laminated Timber (Glulam) and Cross Laminated Timber (CLT) is given in Table 1 .

[Table 1 here]

Dowel-type connections with nails, screws, dowels and bolts are the most commonly used connection type in timber buildings. First introduced by Johansen (1949), the EYM is widely accepted as able to predict the ductile capacity of dowel-type 
connections with reasonable accuracy (Pedersen et al. 1999) and has been adopted by many timber design codes around the world including Eurocode 5 (2004). As an example, Figure 3 shows the possible failure modes defined in the EYM for a steel-totimber connection with a thick steel side plate or an internal steel plate. Equation 2 gives the respective prediction of the ultimate ductile capacity, $F_{u}$, per fastener and shear plane based on the timber thickness, $t$, fastener diameter, $d$, fastener bending moment, $M_{y}$, and timber embedment strength, $f_{h}$, as well as the rope effect, $F_{\text {rope }}$. For bolts and nails, the rope effect is determined as $F_{\text {rope }}=\min \left(F_{a x} / 4,0.25 F_{u}\right)$, where $F_{a x}$ is the axial capacity of the fastener, and is the $F_{u}$ Johansen part. $F_{\text {rope }}$ equals zero for smooth dowels.

$$
F_{u}=\min \left\{\begin{array}{c}
f_{h} t d\left[\sqrt{2+\frac{4 M_{h} t d}{f_{h} d t^{2}}-1}\right]+F_{\text {rope }} \\
2.3 \sqrt{M_{y} f_{h} d}+F_{\text {rope }}
\end{array}\right.
$$

\section{Analytical method to derive overstrength}

Figures $4 \mathrm{a}$ to $4 \mathrm{c}$ show a breakdown of the individual overstrength components that constitute $\gamma_{R d}$ based on the definitions in Equation 1. As the connection strength calculated by the EYM depends on the timber material embedment strength $f_{h}$ (which is correlated to timber density $\rho$ and fastener diameter) and the fastener bending moment $M_{y}$ (which is correlated to fastener yield strength $f_{y}$ and cross section), each overstrength component can be subdivided into a contribution from the timber and steel material, as shown in Figure $4 \mathrm{~b}$ and $4 \mathrm{c}$.

In the following sections a detailed procedure is presented to analytically derive and quantify $\gamma_{\text {mod }}$ and $\gamma_{0.95}$ for dowel-type connections in the context of Eurocode 5 based on 
material properties. In addition, accidental overstrength, $\gamma_{\text {err }}$, is discussed which is introduced when materials with higher than specified grades are supplied.

\section{Material safety factor $\gamma_{M}$}

The material safety factor is defined as $\gamma_{M}=F_{u, k} / F_{u, d}$ and is given in the respective design standards: For dowelled connections in sawn timber, Eurocode 5 (2004) recommends $\gamma_{M}=1.3$, and NZS3603 (1993) recommends a strength reduction factor of $\phi=0.8$ which leads to $\gamma_{M}=1 / \phi=1.25$. In seismic design, however, $\gamma_{M}$ is not considered: NZS3603 (1993) recommends $\phi=1.0$ and Eurocode 8 (2004) recommends $\gamma_{M}=1.0$. Therefore, the total theoretical overstrength is reduced to $\gamma_{R d, t h}=\gamma_{\text {mod }} \gamma_{0.95}$

\section{Material variability within speficied grade $\gamma_{0.95}$}

Jorissen and Fragiacomo (2011) attributed $\gamma_{0.95}=F_{u, 0.95} / F_{u, 0.05}$ to the scatter of the connection strength properties. The $5^{\text {th }}$ and $95^{\text {th }}$-percentiles of the connection strength distribution are directly related to the variability of the timber material and steel fasteners within the specified material grade. It is thus possible to estimate $\gamma_{0.95}$ by inserting the $5^{\text {th }}$ and $95^{\text {th }}$-percentile of the timber density and steel yield strength distributions into the EYM. To determine $\gamma_{0.95}$, either upper and lower bounds of the material grade can be used, although supplier information is preferable as it yields less conservative $\gamma_{0.95}$ values. Timber suppliers generally record density as well as other material properties to determine the material grade. In a similar manner, steel fastener manufacturers conduct testing for each batch and steel grade produced. The method to determine $\gamma_{0.95}$ is shown for the calculation of $F_{u}$ of dowel-type timber connections with parallel-to-grain loading in Equations 3.1 through 3.3, where the embedment strength formulas in Equation 3.2 are given in Eurocode 5 for sawn timber, the CLT Handbook for CLT (FPInnovations 2011), and by Franke and Quenneville (2011) for LVL. The 
resulting overstrength factors then depend on the governing EYM failure mode, which in turn depends on the timber thickness, $t$, fastener diameter, $d$, and material properties. Upper-bound overstrength subcomponents from the timber and steel contributions, $\gamma_{0.95, f h}$ and $\gamma_{0.95, M y}$, are given in Table 2.

$$
\begin{gathered}
\gamma_{0.95}=\gamma_{0.95, f h} \gamma_{0.95, M y} \\
\gamma_{0.95 . f h}=\frac{F\left(f_{h, 0.95}\right)}{F\left(f_{h, 0.05}\right)} \text { where } f_{h, 0.05}=f\left(\rho_{0.05}\right) ; f_{h, 0.95}=f\left(\rho_{0.95}\right) \\
\text { and } f_{h}=\left\{\begin{array}{cc}
0.082(1-0.01 d) \rho & \text { for sawn timber } \\
0.031(1-0.015 d) \rho^{1.16} & \text { for } C L T \\
0.075(1-0.0037 d) \rho & \text { for } L V L
\end{array}\right. \\
\gamma_{0.95 . M y}=\frac{F\left(M_{y, 0.95}\right)}{F\left(M_{y, 0.05}\right)} \text { where } M_{y, 0.05}=f_{y, 0.05} d^{3} / 6 \text { and } M_{y, 0.95}=f_{y, 0.95} d^{3} / 6
\end{gathered}
$$

\section{Conservatism in Models $\gamma_{\bmod }$}

Analytical model overstrength, $\gamma_{\text {mod }}$, quantifies the difference between the $5^{\text {th }}$-percentile of the actual connection strength, $F_{u, 0.05}$, and the characteristic strength, $F_{u, k}$, calculated by the EYM. As shown in Figure 4c, $\gamma_{\text {mod }}$ stems from conservatism in the material models as well as the difference between the characteristic yield strength and nominal yield strength of steel fasteners which is prescribed by design codes. The overstrength in the material models encompasses the conservatism of semi-empirical calculation formulas as well as the approximation error of the analytical formulas used to predict the connection strength (Jorissen and Fragiacomo 2011).

Eurocode 5 (2004) uses the effective bending yield moment $M_{e f f}=0.3 d^{2.6} f_{u}$ to calculate the fastener yield moment, $M_{y}$, based on the assumption that full plasticisation of the cross sections of stocky fasteners is hardly achieved in timber connections (Blaß et al. 2001). However, as Stehn and Johnsson (2002) pointed out, this is due to timber splitting which leads to mode cross-over from a ductile response to brittle failure of the 
joint itself. A detailed explanation of mode cross-over due to reduced brittle resistance is given in Stehn and Johnsson (2002), Novis et al. (2016), and Ottenhaus et al. (2018a). In highly ductile connections, fasteners can achieve full plastic hinges with $M_{y}=M_{p}=f_{y}$ $d^{3} / 6$. If capacity design is used to achieve ductile connection behaviour, the use of $M_{y}=M_{\text {eff }}$ instead of $M_{y}=M_{p}$ can introduce significant model overstrength (Schick et al. 2016), which, depending on the governing EYM mode, can reach up to $\gamma_{\text {mod,Meff }}=1.44$, as shown in Figure 5 for the allowable steel strength range of AS/NZS 4671 (2001). Semi-empirical material models for timber connection design are usually established by conservatively calibrating an analytical model to experimental data. Examples of such models are the different embedment strength formulas given in Equation 3.2. If sufficient data is gathered the difference between calculated and experimental embedment strength can be minimised, which may lead to small overstrength factors $\gamma_{m o d, f h}$

The difference between the $5^{\text {th }}$-percentile of the steel yield strength, $f_{y, 0.05}$, and nominal strength, $f_{y, n o m}$, introduces overstrength as well. Karmazínová and Melcher (2012) conducted experiments on grade S235 and S355 steel (grades according to Eurocode 3) and another set of tensile test data of Grade 300 and Grade 500 steel was made available by a New Zealand steel supplier (grades according to AS/NZS 4671 2001).

Overstrength from nominal steel strength, $\gamma_{\text {mod,nom }}$, can be estimated by inserting $f_{y, 0.05}$ and $f_{y, \text { nom }}$ into the EYM calculations. The resulting upper-bound overstrength values are given in Table 2 .

Finally, it should be noted that larger sample sizes result in smaller overstrength factors $\gamma_{0.95}$ and $\gamma_{\text {mod }}$ and that stress grading reduces the variability in timber material properties and therefore can reduce $\gamma_{0.95}$, as shown in Table 2 .

[Table 2 here] 


\section{Accidental overstrength $\gamma_{\mathrm{err}}$}

Accidental overstrength, $\gamma_{e r r}$, is introduced if the supplied material grade is significantly higher (stronger) than the specified grade due to the common misconception that a stronger material is 'better' (Misconel et al. 2016, Sandhaas et al. 2017). In practice, this mistake often goes unnoticed, since high strength steel is visually no different from mild steel and the error would only be discovered through experimental testing. Installation of the higher strength material can lead to unsafe seismic design since the connection may now no longer be the weakest link the in the strength hierarchy. Accordingly, the accidental overstrength can then be defined as $\gamma_{\text {err }}=F_{\text {supplied }} / F_{\text {specified }}$.

\section{Results of model validation and discussion}

The analytical method is checked against the results of dowelled and nailed hold-down connection tests conducted at structural engineering laboratories of the University of Canterbury. These hold-down connections were designed as highly ductile elements for mass timber shear walls that could develop overstrength and ductile behaviour and thereby qualified as potential ductile elements following the capacity design approach. As suggested by Smith et al. 2006, highly ductile behaviour is defined as $\mu=\Delta_{u} / \Delta_{y}>6$, where $\mu$ is the connection ductility, $\Delta_{u}$ is the ultimate displacement, and $\Delta_{y}$ is the yield displacement. Furthermore, the analytical method is further checked against previous experimental datasets from literature. For a detailed step-by-step procedure and example calculations refer to Ottenhaus et al. $2018 \mathrm{~d}$.

\section{Validation with experimental data from previous testing}

Table 3 gives the material input properties for the characteristic strength prediction, $F_{u, k, p r e d}$, of the connection tests, as well as theoretical overstrength prediction, $\gamma_{R d, t h}$. Various connection layouts with expected ductile behaviour were subjected to 
monotonic and cyclic loading (ISO and CUREE loading protocols for nailed connections and ISO loading for dowelled connections as described in Ottenhaus et al. 2016).

The nailed hold-down connections consisted of fifteen $ø 4 \times 100 \mathrm{~mm}$ nails and $10 \mathrm{~mm}$ thick steel side plates. The nails were spaced densely in LVL ( $4 d$ fastener spacing parallel to grain and $3 d$ perpendicular to grain) and widely in CLT ( $8 d$ fastener spacing parallel to grain and $6 d$ perpendicular to grain). Nail bending tests were conducted to determine the nail yield moment from which the yield strength was derived as $f_{y}=$ $530 \mathrm{MPa}$. More information on the nailed hold-down connection tests can be found in Ottenhaus et al. 2016.

The dowelled hold-down connections consisted of an internal steel plate and ø20 $\mathrm{mm}$ dowels with specified Grade 300 steel. The small-scale tests were conducted in CLT and LVL with varying fastener spacing and the large-scale test were conducted in CLT and a CLT-LVL layup. More information regarding the dowelled connection test setups can be found in Ottenhaus et al. 2018a and 2018c.

It was found that EYM mode iii in Equation 2 was governing for all nailed hold-downs and mode ii was the governing case for all dowelled hold-downs.

Overstrength from the material strength distribution within the designated grade, $\gamma_{0.95}=$ $F_{0.95} / F_{0.05}$ was calculated using the EYM and Equations 3.2 and 3.3. and data from Table 2.

Model overstrength, $\gamma_{\text {mod }}=F_{0.05} / F_{k}$ was estimated using the EYM by inserting $5^{\text {th }}$ percentile timber density, $\rho_{0.05, \exp }$, into embedment strength formulas (Equation 3.2), and by contrasting the results with experimental data from Ottenhaus and $\mathrm{Li}$ (2018). In addition, nominal fastener yield strength was compared to $5^{\text {th }}$-percentile yield strength. 
It was found that $\gamma_{\text {mod }}$ is relatively low (around 10\%). Note that for the nailed connections, no embedment tests were conducted and $\gamma_{\text {mod }}$ was estimated based on the results for dowelled small-scale connections.

For the large-scale connection tests, Grade 500 dowels instead of Grade 300 dowels were supplied which was discovered during tensile and three-point bending tests (Ottenhaus et al. 2018b), which means the overstrength estimates were nonconservative. The error can be expressed as accidental overstrength $\gamma_{\text {err }}=F\left(f_{y}=500 \mathrm{MPa}\right)$ / $F\left(f_{y}=300 \mathrm{MPa}\right)$. Note that accidental overstrength cannot be predicted or accounted for in practice unless experimental testing is carried out. In consequence, additional overstrength due to supply of a stronger steel grade can jeopardise seismic safety and cause premature brittle failure since the ductile connection response may now no longer be the weakest link in the strength hierarchy. Therefore, it is important raise awareness of this issue in the professional community and to educate suppliers on capacity design.

A summary of the resulting theoretical overstrength and its components is given in Table 3. In those cases where no experimental data was available, a conservative assumption was made.

[Table 3 here]

Table 4 gives the various connection tests results as well as ductility, $\mu$, experimental overstrength, $\gamma_{R d, \exp }=F_{\text {max }, \text { exp }} / F_{u, k, p r e d}$, and theoretical overstrength, $\gamma_{R d, t h}=\gamma_{0.95} \gamma_{\text {mod }}$. The cases where the overstrength estimation was non-conservative $\left(\gamma_{R d, \exp }>\gamma_{R d, t h}\right)$ are printed in bold. For the small-scale dowelled connections tests this was due to a specimen that exhibited exceptional strength. For the large-scale dowelled connection tests this was due to accidental overstrength and when $\gamma_{\text {err }}$ was considered, the 
overstrength prediction was conservative. Note that the estimated overstrength values for dowels are smaller than the previously proposed overstrength factor of 1.68 (Ottenhaus et al. 2017), which was conservatively based on upper-bound overstrength component values that did not take the governing EYM mode into account.

[Table 4 here]

Figure 6 visualises some of the data presented in Table 4 and displays the load displacement and backbone curves as well as predicted strengths for the small-scale nailed and dowelled hold-down connection tests. As can be seen from Table 4 and Figure 6, the overstrength estimation yielded conservative results in most cases and generally agreed well with the experimental data $\left(\max \gamma_{R d, \exp } \approx \gamma_{R d, t h}\right.$, and $\gamma_{R d, t h}$ was on average $18 \%$ conservative and non-conservative in one case), except when the wrong steel grade was supplied.

\section{Validation with experimental data from literature}

The previously described method was validated with experimental data from literature. The studies were selected based on the following criteria:

- failure was truly ductile (i.e. $\mu>4$ )

- data regarding density distribution and steel material grade was either included in the publication or made available

- connection geometry was described sufficiently well to estimate capacity

- load displacement curves were made available to estimate ductility $\mu$

Jorissen (1998) conducted experiments on symmetric bolted timber-to-timber connections in sawn European Spruce. The slenderness ratio of the connection specimens was varied by changing the timber member thicknesses of the outer 
members, $t_{1}$, and inner member, $t_{2}$. The average timber density and standard deviation are given in Jorissen's thesis as $\rho_{a v}=450 \mathrm{~kg} / \mathrm{m}^{3}$ and $\sigma=44 \mathrm{~kg} / \mathrm{m}^{3}$, respectively. The bolts were specified M12 $(d=12 \mathrm{~mm})$ grade 4.6 with a nominal yield strength of $f_{y, n o m}=$ $240 \mathrm{MPa}$ (DIN931 1987). An upper-bound for the yield strength was estimated as $f_{y, 0.95}$ $=300 \mathrm{MPa}$ based on the allowable range given for mild steel in AS/NZS 4671 (2001) as this is the only commonly used material standard that specifies an upper-bound strength for mild steel. Table 5 gives the experimental strength, predicted strength, experimental overstrength, ductility, as well as predicted overstrength for those test series that responded in a ductile $(\mu>4)$ or highly ductile $(\mu>6)$ manner and produced overstrength, with a conservative assumption of $\gamma_{m o d}=1.15$. It should be noted that the predicted strength values in Table 5 may differ from those in Jorisson 1998 since the plastic yield moment was used for the strength prediction in this paper. The cases where the overstrength estimation was non-conservative $\left(\gamma_{R d, \exp }>\gamma_{R d, t h}\right)$ are printed in bold. [Table 5 here]

Dorn et al. (2013) conducted experiments on single dowel connections in double shear with an internal steel plate. The dowel diameter was $d=12 \mathrm{~mm}$ with varying timber thickness, $t$. According to Dorn et al., the widths of the specimens were chosen in order to produce one of the three main ductile responses in Eurocode 5 (shown in Figure 3b) and it was found that the ductility increases with the width of the specimen. The timber species was Norwegian Spruce and the density percentiles were based on the values given in the publication with assumed grade $\mathrm{C} 24: \rho_{0.05}=350 \mathrm{~kg} / \mathrm{m}^{3}$ and $\rho_{0.95}=510$ $\mathrm{kg} / \mathrm{m}^{3}$. In the publication only a value for $f_{u}=708 \mathrm{MPa}$ was given and hence an 'educated guess' had to be made regarding the nominal steel strength and $95^{\text {th }}$-percentile strength: $f_{y, \text { nom }}=500 \mathrm{MPa}$ and $f_{y, 0.95}=625 \mathrm{MPa}$, based on the allowable ranges for $f_{y}$ as well as $f_{u} / f_{y}$ ratios given in other material standards. The maximum experimental 
strength values were derived from the graphs provided in the publication. The ductility was estimated based on the graphs as ductile $(\mathrm{D}, \mu>4)$ or highly ductile (HD, $\mu>6)$. The results are given in Table 6 and those cases where the predicted overstrength was non-conservative are printed in bold.

[Table 6 here]

As can be seen from Tables 8 and 9, the overstrength factors derived by the analytical method generally agreed well with the experimental data $\left(\max \gamma_{R d, \exp } \approx \gamma_{R d, t h}\right.$, and $\gamma_{R d, t h}$ was on average $23 \%$ conservative and non-conservative in two cases).

\section{Conclusions}

This paper presented an analytical method to derive overstrength factors of dowel-type timber connections that are commonly designed as potential ductile elements in timber structures following the capacity design approach. The analytical derivation is based on ductile failure modes in the European yield model considering the variability of material properties and conservatism in semi-empirical embedment and dowel yield moment models. The material properties used in the analytical method are either specified by strength classes in design codes or obtained from steel and timber suppliers.

Experimental data of nailed and dowelled hold-down connections that are designed as ductile elements in mass timber shear walls as well as additional connection test data in literature were used to check the accuracy of the analytical method. It was found that the analytical overstrength factors generally agreed well with the test results and were slightly conservative in most cases, except when the supplied steel yield strength well exceeded that of the specified steel grade. 
In general, this method provides reasonably accurate estimations of the upper-bound overstrength for dowel-type connections made of bolts, dowels, and nails, so that it has the potential to replace costly experimental derivation of connection overstrength. Provided accurate material models such as the ones presented in this paper are used for embedment strength and fastener strength calculations (i.e. use plastic fastener bending moment $M_{p}$ rather than effective yield moment $M_{\text {eff }}$ ), the only required input data is the timber and steel material grade. However, where possible supplier information should be used to reduce conservatism in the overstrength estimation to yield a more economic design. This method therefore can provide designers with a relatively simple tool to calculate overstrength factors for specific ductile connection layouts without making overly conservative assumptions in capacity design.

It should also be noted that while the presented method is independent of material, fastener type, and connection layout, a good understanding of conservatism in the underlying analytical models is necessary to apply the method to other fastener types such as screws and rivets. In future research, the methodology may thus be extended to and validated for different fasteners types, connection layouts, loading scenarios, and timber materials. 


\section{Word count main text 3644}

Word count abstract 100

\section{Acknowledgements}

The research was funded jointly by Natural Hazards Research Platform, Earthquake Commission (EQC) of New Zealand, and ARC Research Hub for Advanced Solutions to Transform Tall Timber Buildings in Australia. The material was provided by XLam NZ Ltd and Nelson Pine Industries Ltd and their support is gratefully acknowledged.

\section{References}

AS/NSZ (Standard Australia, Standards New Zealand) (2005). "Structural Laminated Veneer Lumber." AS/NZS 4357.0:2005, Sydney, Wellington.

AS/NSZ (Standard Australia, Standards New Zealand) (2001). "Steel reinforcing materials." AS/NZS 4671:2001, Sydney, Wellington.

Blaß, H. J., Bienhaus, A., and Krämer, V. (2001). "Effective bending capacity of dowel-type fasteners." Proceedings of the International RILEM Symposium Joints in Timber Structures, Stuttgart, 22, 71-88.

Brühl, F., Schänzlin, J., and Kuhlmann, U. (2014). "Ductility in Timber Structures: Investigations on Over-Strength Factors." RILEM Bookseries (Vol. 9) Springer Netherlands, Dordrecht.

DIN (Deutsches Institut fur Normung ) (1987). "Sechskantschauben mit Schaft, Gewine M 1.6 bis M39, Produktklassen A und B.” DIN931 Teil 1, Berlin.

Dorn, M., de Borst, K., and Eberhardsteiner, J. (2013). "Experiments on dowel-type timber connections." Engineering Structures, 47, 67-80.

CEN (European Committee for Standardization) (2005). "Design of steel structures, Part 1.1." Eurocode 3, Brussels

CEN (European Committee for Standardization) (2004). "Design of timber structures, Part 1.1." Eurocode 5, Brussels

CEN (European Committee for Standardization) (2004). "Design of structures for earthquake resistance, Part 1." Eurocode 8, Brussels

CEN (European Committee for Standardization) (2016). "Structural timber. Strength classes." EN338:2016, Brussels. 
Fardis, M. N. (2018). Capacity design: Early history. Earthquake Engineering and Structural Dynamics, 47(14), 2887-2896.

Follesa, M., Fragiacomo, M., \& Lauriola, M. (2011). A proposal for revision of the current timber part (Section 8) of Eurocode 8 Part. Proceedings of CIB-W18 meeting forty-four, Alghero, Italy, 2011.

Follesa, M., Fragiacomo, M., Casagrande, D., Tomasi, R., Piazza, M., Vassallo, D., ... Rossi, S. (2018). The new provisions for the seismic design of timber buildings in Europe. Engineering Structures, 168(December 2017), 736-747.

FPInnovations (2011). CLT Handbook. E. Karacabeyli and R. Desjardins, Eds.

Fragiacomo, M., Dujic, B., and Sustersic, I. (2011). "Elastic and ductile design of multi-storey crosslam massive wooden buildings under seismic actions." Engineering Structures, 33(11), 3043-3053.

Franke, S., and Quenneville, P. (2011). "Bolted and dowelled connections in radiate pine and laminated veneer lumber using the European yield model." Australian Journal of Structural Engineering, 12(1), 13-28.

Gavric, I., Fragiacomo, M., and Ceccotti, A. (2014). "Cyclic behaviour of typical metal connectors for cross-laminated (CLT) structures.” Materials and Structures, 48(6), 1841-185.

Gavric, I., Fragiacomo, M., and Ceccotti, A. (2015). "Cyclic behavior of typical screwed connections for cross-laminated (CLT) structures." European Journal of Wood and Wood Products, 73(2), 179-191.

Izzi, M., Flatscher, G., Fragiacomo, M., \& Schickhofer, G. (2016). “Experimental investigations and design provisions of steel-to-timber joints with annular-ringed shank nails for CrossLaminated Timber structures." Construction \& building materials, 122, 446-457.

Johansen, K. W. (1949). “Theory of Timber Connections.” Int Assoc Bridge and Struct Eng, 9, 249-262.

Jorissen, A. (1998). "Double shear timber connections with dowel type fasteners." PhD thesis, TU Delft.

Jorissen, A., and Fragiacomo, M. (2011). "General notes on ductility in timber structures." Engineering Structures, 33(11), 2987-2997.

Karmazínová, M., and Melcher, J. (2012). "Influence of Steel Yield Strength Value on Structural Reliability of Steel Yield Strength." Recent Researches in Environmental and Geological Sciences, 2012, 441-446.

Loss, C., Zonta, D., \& Piazza, M. (2013). On Estimating the Seismic Displacement Capacity of Timber Portal-Frames. Journal of Earthquake Engineering, 17(6), 879-901. 
Misconel, A., and Ballerini, M. (2016). "Steel-To-Timber Joints of Beech-LVL With Very High Strength Steel Dowels". Proceedings of World Conference on Timber Engineering (WCTE2016), Vienna.

Moroder, D., Smith, T., Pampanin, S., Palermo, A., and Buchanan, A. H. (2014). "Design of Floor Diaphragms in Multi-Storey Timber Buildings". Proceedings of International Network on Timber Engineering Research Meeting, 2014, Bath.

Novis, S. A. L., Jacks, J., \& Quenneville, P. (2016). Predicting the Resistance and Displacement of Timber Bolted Connections. Proceedings of World Conference on Timber Engineering (WCTE2016), Vienna.

NZS (Standards New Zealand). (1993). “Timber Structures Standard.” NZS3603:1993, Wellington.

O'Ceallaigh, C., \& Harte, A. M. (2019). "The elastic and ductile behaviour of CLT wall-floor connections and the influence of fastener length." Engineering Structures, 189(March), 319331 .

Ottenhaus, L.-M. (2019). "theoretical overstrength." Figshare (Figure). doi:

$10.6084 / \mathrm{m} 9$. figshare.7834736v3

Ottenhaus, L.-M. (2019a). “experimental overstrength.” Figshare (Figure). doi:

$10.6084 / \mathrm{m} 9$. figshare.7834751v2

Ottenhaus, L.-M. (2019b). “Overstrength Components.” Figshare (Figure). doi:

10.6084/m9.figshare.8239892.v1

Ottenhaus, L.-M. (2019a). “Overstrength from material variability.” Figshare (Figure). doi: 10.6084/m9.figshare.8239898.v1

Ottenhaus, L.-M. (2019d). “Overstrength from model conservatism.” Figshare (Figure). doi: 10.6084/m9.figshare.8239901.v2

Ottenhaus, L.-M., Li, M., Smith, T., \& Quenneville, P. (2016). Ductility of dowelled and nailed CLT and LVL connections under monotonic and cyclic loading. Australian Earthquake Engineering Society 2016 Conference.

Ottenhaus, L.-M., and Li, M. (2018). "Embedment Strength of New Zealand Cross Laminated Timber." New Zealand Timber Design Journal, 26(1), 12-16.

Ottenhaus, L.-M., Li, M., Smith, T., and Quenneville, P. (2018a). "Mode Cross-Over and Ductility of Dowelled LVL and CLT Connections under Monotonic and Cyclic Loading." Journal of Structural Engineering, 144(7), 1-10.

Ottenhaus, L.-M., Li, M., and Smith, T. (2018b). "Overstrength of large-scale dowelled connections in CLT." Proceedings of NZSEE Conference, 2018, Auckland. 
Ottenhaus, L.-M., Li, M., and Smith, T. (2018c). "Structural performance of large-scale dowelled CLT connections under monotonic and cyclic loading." Engineering Structures, 176(August), 41-48.

Ottenhaus, L.-M., Li, M., and Smith, T. (2018d). "Analytical Method to Derive Overstrength of Dowel-Type Connections." Proceedings of International Network on Timber Engineering Research Meeting, 2018, Tallinn.

Ottenhaus, L.-M., Li, M., Smith, T., and Quenneville, P. (2017). "Overstrength of dowelled CLT connections under monotonic and cyclic loading." Bulletin of Earthquake Engineering.

Park, R., and Paulay, T. (1975). "Reinforced Concrete Structures." Hoboken, NJ, USA: John Wiley \& Sons.

Pedersen, M. U., Clorius, C. O., Damklide, L., Hoffmeyer, P., and Eskildsen, L. (1999). "Dowel type connections with slotted-in steel plates." Proceedings of CIB-W18 meeting thirty-two, Graz, Austria, 1999.

Popovski, M., Prion, H. G., and Karacabeyli, E. (2002). "Seismic performance of connections in heavy timber construction." Canadian Journal of Civil Engineering, 29(3), 389-399.

Sandhaas, C., and van de Kuilen, J. W. G. (2017). "Strength and stiffness of timber joints with very high strength steel dowels." Engineering Structures, 131, 394-404.

Schick, M., Seim, W., and Kruger, S. (2016). "Dowel-Type Fasteners with Small Diameters Review and Improvement of Design Formulations." Proceedings of World Conference on Timber Engineering (WCTE2016), Vienna.

Schick, M., Vogt, T., and Seim, W. (2013). "Connections and anchoring for wall and slab elements in seismic design." Proceedings of CIB-W18 meeting, Vancouver, Canada, 2013, 31332 .

Smith, I., Asiz, A., Snow, M., \& Chui, Y. H. (2006). Possible Canadian / ISO Approach to Deriving Design Values From Test Data. Proceedings of CIB-W18 meeting thirty-nine

Stehn, L., and Johnsson, H. (2002). "Ductility Aspects in Nailed Glue Laminated Timber Connection Design.” Journal of Structural Engineering, 128(3).

Trutalli, D., Marchi, L., Scotta, R., \& Pozza, L. (2018). "Capacity design of traditional and innovative ductile connections for earthquake-resistant CLT structures." Bulletin of Earthquake Engineering

Vogt, T., and Seim, W. (2014). "Advanced modelling of timber-framed wall elements for application in engineering practice." Proceedings of International Network on Timber Engineering Research Meeting, Bath, 2014. 


\section{Notations}

The following symbols are used in this paper:

\begin{tabular}{|c|c|}
\hline$d$ & fastener diameter $\varnothing$ \\
\hline$F_{0.05}$ & $5^{\text {th }}$-percentile of the strength distribution \\
\hline$F_{0.95}$ & $95^{\text {th }}$-percentile of the strength distribution \\
\hline$F_{a x}$ & axial strength used to determine the rope effect $F_{\text {rope }}$ \\
\hline$F_{B R}$ & brittle strength \\
\hline$f_{\text {calc }}$ & calculated strength \\
\hline$F_{d}$ & design strength \\
\hline$F_{E, d}$ & design demand \\
\hline$F_{\text {exp }}$ & experimentally measured strength \\
\hline$f_{h}$ & timber embedment strength \\
\hline$F_{k}$ & characteristic strength \\
\hline$F_{\max }$ & maximum strength \\
\hline$f_{\text {nom }}$ & nominal strength \\
\hline$F_{\text {pred }}$ & predicted strength \\
\hline$F_{\text {rope }}$ & rope effect \\
\hline$F_{\text {specified }}$ & specified strength \\
\hline$F_{\text {supplied }}$ & supplied strength \\
\hline$F_{u}$ & ultimate ductile strength \\
\hline$f_{u}$ & fastener ultimate tensile strength \\
\hline$f_{y}$ & fastener yield strength \\
\hline$f_{y, \text { nom }}$ & nominal fastener yield strength \\
\hline$M_{y}$ & fastener bending moment \\
\hline$M_{e f f}$ & effective fastener bending moment \\
\hline$M_{p}$ & plastic fastener bending moment \\
\hline$t$ & timber member thickness \\
\hline$\gamma_{0.95}$ & overstrength factor to account for material strength distribution \\
\hline$\gamma_{0.95, f h}$ & overstrength from embedment strength distribution \\
\hline$\gamma_{0.95, M y}$ & overstrength from fastener yield strength distribution \\
\hline$\gamma_{e r r}$ & accidental overstrength $\gamma_{\text {err }}=F_{\text {supplied }} / F_{\text {specified }}$ \\
\hline$\gamma_{M}$ & partial material safety factor \\
\hline$\gamma_{\text {mod }}$ & overstrength factor to account for conservatism of analytical models \\
\hline$\gamma_{\text {mod,fh }}$ & overstrength from conservatism in embedment strength models \\
\hline$\gamma_{\text {mod,Meff }}$ & overstrength from using $M_{\text {eff }}$ rather than $M_{p}$ \\
\hline$\gamma_{\text {mod,nom }}$ & overstrength from nominal fastener yield strength \\
\hline$\gamma_{R d}$ & overstrength factor \\
\hline$\gamma_{R d, \text { exp }}$ & experimentally established overstrength factor $\gamma_{R d, \text { exp }}=F_{\max , \exp } / F_{u, k, p r e d}$ \\
\hline$\gamma_{R d, t h}$ & theoretical connection overstrength factor $\gamma_{R d, t h}=\gamma_{\bmod } \gamma_{0.95}$ \\
\hline$\Delta_{u}$ & ultimate displacement \\
\hline$\Delta y$ & yield displacement \\
\hline$\mu$ & ductility $\mu=\Delta_{u} / \Delta_{y}$ \\
\hline$\rho$ & timber density \\
\hline$\rho_{a v}$ & average timber density \\
\hline
\end{tabular}


$\sigma \quad$ standard deviation

$\phi \quad$ strength reduction factor 


\section{Tables}

Table1. Overstrength values in literature for different timber connections.

\begin{tabular}{|c|c|c|c|c|c|}
\hline Author & Connection type & $\gamma_{\text {mod }}$ & $\gamma_{0.95}$ & $\gamma_{R d, t h}$ & $\gamma_{R d, \exp }$ \\
\hline NZS3603 (1993) & nailed connections, sawn & & & 1.6 & \\
\hline Popovski et al. (2002) & bolted connections, Glulam & & & & $1.5-2.0$ \\
\hline $\begin{array}{l}\text { Fragiacomo et al. } \\
(2011)\end{array}$ & nailed hold-downs, CLT & & & & 1.3 \\
\hline Schick et al. (2013) & nailed hold-downs, CLT & $1.0-1.8$ & $1.0-1.4$ & $1.1-2.5$ & \\
\hline Gavric et al. (2014) & nailed hold-downs, CLT & $1.3-2.8$ & $1.2-1.4$ & $1.5-4.0$ & \\
\hline Vogt et al. (2014) & nailed hold-downs, CLT & 1.3 & 1.3 & 1.7 & \\
\hline \multirow{5}{*}{$\begin{array}{l}\text { Gavric et al. (2015) } \\
\text { Izzi et al. (2016) }\end{array}$} & screwed connections, CLT & & & & 1.6 \\
\hline & annular-ringed shank nails, & & & & \\
\hline & CLT, withdrawal & 1.13 & 1.76 & 2.0 & 1.8 \\
\hline & lateral load parallel to grain & 1.44 & 1.27 & 1.8 & 1.3 \\
\hline & lateral load perp. to grain & 1.48 & 1.53 & 2.3 & 1.5 \\
\hline O'Ceallaigh and Harte & screwed brackets, CLT (C16) & & & & \\
\hline \multirow[t]{2}{*}{ (2019) } & tension & $1.1-1.4$ & $1.5-1.9$ & 2.5 & \\
\hline & shear & $1.1-1.3$ & $1.2-1.5$ & 2.1 & \\
\hline
\end{tabular}


Table 2. Upper-bound embedment-related material overstrength $\gamma_{0.95, f h}$ from timber density distribution, upper-bound fastener-related material overstrength $\gamma_{0.95, M y}$ from steel yield strength distribution and allowable range, and upper-bound model overstrength $\gamma_{\text {mod,nom }}$ from nominal steel strength.

\begin{tabular}{|c|c|c|c|c|}
\hline & sample size & $\rho_{0.05}\left[\mathrm{~kg} / \mathrm{m}^{3}\right]$ & $\rho_{0.95}\left[\mathrm{~kg} / \mathrm{m}^{3}\right]$ & $\gamma_{0.95, f h}{ }^{\mathrm{d})}$ \\
\hline ungraded Radiata pine ${ }^{\text {a) }}$ & $\mathrm{n} / \mathrm{a}$ & 402 & 608 & 1.51 \\
\hline LVL11 b) & 57 & 480 & 564 & 1.18 \\
\hline LVL13 b) & 67 & 500 & 585 & 1.17 \\
\hline $\mathrm{C} 18^{\mathrm{c})}$ & $\mathrm{n} / \mathrm{a}$ & 320 & 440 & 1.38 \\
\hline \multirow[t]{2}{*}{$\mathrm{C} 24^{\mathrm{c})}$} & $\mathrm{n} / \mathrm{a}$ & 350 & 490 & 1.40 \\
\hline & sample size & $f_{y, 0.05}\left[\mathrm{~kg} / \mathrm{m}^{3}\right]$ & $f_{y, 0.95}\left[\mathrm{~kg} / \mathrm{m}^{3}\right]$ & $\gamma_{0.95, M y}{ }^{\mathrm{d})}$ \\
\hline $\mathrm{S} 235^{\mathrm{e})}$ & 26 & 284 & 377 & 1.15 \\
\hline $\mathrm{S} 355$ e) & 19 & 410 & 498 & 1.10 \\
\hline Grade 300 f) & $\mathrm{n} / \mathrm{a}$ & 300 & 380 & 1.13 \\
\hline \multirow[t]{2}{*}{ Grade 500 f) } & $\mathrm{n} / \mathrm{a}$ & 500 & 600 & 1.10 \\
\hline & sample size & $f_{y, \text { nom }}\left[\mathrm{kg} / \mathrm{m}^{3}\right]$ & $f_{y, 0.05}\left[\mathrm{~kg} / \mathrm{m}^{3}\right]$ & $\gamma_{\text {mod,nom }}{ }^{\mathrm{d})}$ \\
\hline $\mathrm{S} 235^{\mathrm{e})}$ & 26 & 235 & 284 & 1.10 \\
\hline $\mathrm{S} 355^{\text {e) }}$ & 19 & 355 & 410 & 1.08 \\
\hline
\end{tabular}


Grade 300 g)

320

320

Grade $500^{\text {g) }}$

500

523

1.02

a) provided by XLam NZ Ltd, ${ }^{\text {b) }}$ provided by Nelson Pine Industries Ltd with grades

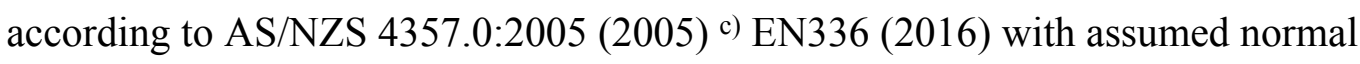
distribution, ${ }^{\text {d) }}$ holds for $\rho \in[300,600] \mathrm{kg} / \mathrm{m}^{3}, d \in[6,30] \mathrm{mm}, t / d \in[2,10]$, e) Karmazínová and Melcher (2012), ${ }^{\text {f) }}$ allowable range AS/NZS 4671:2001 (2001), g) tensile test data provided by Pacific Steel. 
Table 3. Material input properties, strength predictions and theoretical overstrength.

\begin{tabular}{|c|c|c|c|c|c|c|c|}
\hline & \multicolumn{5}{|c|}{ small-scale hold-downs } & \multicolumn{2}{|c|}{ large-scale hold-downs } \\
\hline & & nails & nails & dowels & dowels & dowels & dowels \\
\hline property & & CLT & LVL & CLT & LVL & CLT & CLT-LVL \\
\hline$\rho_{k}$ & {$\left[\mathrm{~kg} / \mathrm{m}^{3}\right]$} & 400 & 500 & 400 & 500 & 400 & $400-480$ \\
\hline$d$ & {$[\mathrm{~mm}]$} & 4 & 4 & 20 & 20 & 20 & 20 \\
\hline$t_{1}\left(L_{p}\right)$ & {$[\mathrm{mm}]$} & 90 & 90 & 55 & 56.5 & 85 & $83(40-43)$ \\
\hline$f_{h, k}$ & [MPa] & 30.4 & 39.4 & 22.6 & 34.7 & 22.6 & 28.2 \\
\hline$f_{y, n o m}$ & {$[\mathrm{MPa}]$} & 530 & 530 & 300 & 300 & 300 & 300 \\
\hline$M_{y}$ & {$[\mathrm{Nm}]$} & 5.7 & 5.7 & 400 & 400 & 400 & 400 \\
\hline$n$ & fasteners & 15 & 15 & 4 & 4 & 16 & 16 \\
\hline$F_{\text {rope }}$ & {$[\mathrm{kN}]$} & $25 \%$ a) & $25 \%$ a) & 0 b) & $0^{b)}$ & $2 \times 25 \% c)$ & $2 \times 25 \% c)$ \\
\hline$F_{u, k, p r e d}$ & {$[\mathrm{kN}]$} & 71 & 81 & 155 & 204 & 734 & 854 \\
\hline$F_{u, 0.05}$ & {$[\mathrm{kN}]$} & 71 & 81 & 160 & 209 & 750 & 879 \\
\hline$F_{u, 0.95}$ & {$[\mathrm{kN}]$} & 104 & 103 & 224 & 240 & 1106 & 1135 \\
\hline$\gamma_{0.95}$ & {$[-]$} & 1.46 & 1.27 & 1.40 & 1.15 & 1.48 & 1.29 \\
\hline$f_{h, k, c a l c}$ & {$[\mathrm{MPa}]$} & 30.4 & 39.4 & 24.4 & - & 24.0 & 28.2 \\
\hline
\end{tabular}




\begin{tabular}{llllllll}
$f_{h, 0.05, \exp }$ & {$[\mathrm{MPa}]$} & - & - & 26.2 & - & 25.1 & 32.1 \\
$f_{y, \text { nom }}$ & {$[\mathrm{MPa}]$} & - & - & 300 & 300 & 300 & 300 \\
$f_{y, 0.05}$ & {$[\mathrm{MPa}]$} & 530 & 530 & 320 & 320 & 320 & 320 \\
$\gamma_{\text {mod }}$ & {$[-]$} & $1.05^{\mathrm{e})}$ & 1.05 e) & 1.05 & $1.05^{\mathrm{a}}$ & 1.07 & 1.13 \\
& & & & & & & \\
$\gamma_{\text {Rd,th }}$ & {$[-]$} & $\mathbf{1 . 5 3}$ & $\mathbf{1 . 3 3}$ & $\mathbf{1 . 4 7}$ & $\mathbf{1 . 2 1}$ & $\mathbf{1 . 5 8}$ & $\mathbf{1 . 4 5}$ \\
& & & & & & & \\
$\gamma_{\text {err }}$ & {$[-]$} & $\mathrm{n} / \mathrm{a}$ & $\mathrm{n} / \mathrm{a}$ & $\mathrm{n} / \mathrm{a}$ & $\mathrm{n} / \mathrm{a}$ & 1.17 & 1.15 \\
\hline
\end{tabular}

a) rope effect nails: $F_{\text {rope }}=\min \left(F_{a x} / 4,0.25 F_{u, k}\right)$ b) all smooth dowels ${ }^{c)} 2$ dowels with threaded ends and nuts and washers in end row, ${ }^{\text {e) }}$ conservative assumption based on other results, $\mathrm{n} / \mathrm{a}=$ not applicable. 
Table 4. Experimental results and validation of $\gamma_{R d, t h}$.

\begin{tabular}{|c|c|c|c|c|c|c|}
\hline & $F_{\max , \exp }[\mathrm{kN}]$ & $F_{u, k, p r e d}[\mathrm{kN}]$ & $\mu[-]$ & $\gamma_{R d, \exp }[-]$ & $\gamma_{R d, t h}[-]$ & $\gamma_{R d, t h} \gamma_{\text {err }}[-]$ \\
\hline \multicolumn{7}{|c|}{ Nailed hold-downs in CLT - wide (Ottenhaus et al. 2016) } \\
\hline MON01 & 88.6 & 71 & 11.1 & 1.24 & 1.53 & $\mathrm{n} / \mathrm{a}$ \\
\hline MON02 & 96.4 & 71 & 8.4 & 1.35 & 1.53 & $\mathrm{n} / \mathrm{a}$ \\
\hline MON03 & 93.9 & 71 & 6.9 & 1.32 & 1.53 & $\mathrm{n} / \mathrm{a}$ \\
\hline ISO01 & 96.9 & 71 & 7.7 & 1.36 & 1.53 & $\mathrm{n} / \mathrm{a}$ \\
\hline ISO02 & 88.1 & 71 & 6.2 & 1.24 & 1.53 & $\mathrm{n} / \mathrm{a}$ \\
\hline ISO03 & 94.2 & 71 & 6.7 & 1.32 & 1.53 & $\mathrm{n} / \mathrm{a}$ \\
\hline CUREE04 & 86.1 & 71 & 6.7 & 1.21 & 1.53 & $\mathrm{n} / \mathrm{a}$ \\
\hline
\end{tabular}

Nailed hold-downs in LVL - dense (Ottenhaus et al. 2016)

\begin{tabular}{lcccccc}
\hline MON01 & 89.5 & 81 & 8.3 & 1.10 & 1.33 & n/a \\
MON02 & 97.1 & 81 & 10.5 & 1.19 & 1.33 & n/a \\
MON03 & 88.1 & 81 & 11.8 & 1.08 & 1.33 & n/a \\
& & & & & & \\
ISO02 & 101.4 & 81 & 10.8 & 1.25 & 1.33 & n/a \\
& & & & & & \\
ISO03 & 88.2 & 81 & 9.1 & 1.08 & 1.33 & $\mathrm{n} / \mathrm{a}$ \\
CUREE01 & 90.7 & 81 & 10.4 & 1.11 & 1.33 & $\mathrm{n} / \mathrm{a}$
\end{tabular}




$\begin{array}{llccccc}\text { CUREE02 } & 102.8 & 81 & 8.7 & 1.26 & 1.33 & \mathrm{n} / \mathrm{a} \\ & & & & & & \\ \text { CUREE03 } & 99.5 & 81 & 6.8 & 1.22 & 1.33 & \mathrm{n} / \mathrm{a}\end{array}$

Small-scale dowelled hold-downs in CLT (Ottenhaus et al. 2017, 2018a)

\begin{tabular}{|c|c|c|c|c|c|c|}
\hline L1-M01 & 195 & 155.4 & 20.8 & 1.25 & 1.47 & $\mathrm{n} / \mathrm{a}$ \\
\hline L1-M03 & 194 & 155.4 & 7.5 & 1.25 & 1.47 & $\mathrm{n} / \mathrm{a}$ \\
\hline L1-M06 & 242 & 155.4 & 41.8 & 1.56 & 1.47 & $\mathrm{n} / \mathrm{a}$ \\
\hline L1-C01 & 193 & 155.4 & 10.4 & 1.24 & 1.47 & $\mathrm{n} / \mathrm{a}$ \\
\hline L1-C02 & 195 & 155.4 & 14.4 & 1.25 & 1.47 & $\mathrm{n} / \mathrm{a}$ \\
\hline L1-C04 & 171 & 155.4 & 7.9 & 1.10 & 1.47 & $\mathrm{n} / \mathrm{a}$ \\
\hline L1-C05 & 162 & 155.4 & 15.4 & 1.04 & 1.47 & $\mathrm{n} / \mathrm{a}$ \\
\hline L2-M01 & 224 & 155.4 & 24.4 & 1.44 & 1.47 & $\mathrm{n} / \mathrm{a}$ \\
\hline L2-M02 & 203 & 155.4 & 17.9 & 1.31 & 1.47 & $\mathrm{n} / \mathrm{a}$ \\
\hline L2-M04 & 194 & 155.4 & 24.5 & 1.25 & 1.47 & $\mathrm{n} / \mathrm{a}$ \\
\hline L3-M02 & 168 & 155.4 & 9.0 & 1.08 & 1.47 & $\mathrm{n} / \mathrm{a}$ \\
\hline L3-M03 & 164 & 155.4 & 9.5 & 1.06 & 1.47 & $\mathrm{n} / \mathrm{a}$ \\
\hline L3-M04 & 187 & 155.4 & 9.3 & 1.20 & 1.47 & $\mathrm{n} / \mathrm{a}$ \\
\hline L3-M05 & 186 & 155.4 & 6.5 & 1.20 & 1.47 & $\mathrm{n} / \mathrm{a}$ \\
\hline
\end{tabular}


Small-scale dowelled hold-downs in LVL (Ottenhaus et al. 2017, 2018a)

\begin{tabular}{lcccccc}
\hline L1-M04 & 210 & 204.0 & 10.0 & 1.03 & 1.21 & $\mathrm{n} / \mathrm{a}$ \\
L1-C01 & 205 & 204.0 & 9.7 & 1.01 & 1.21 & $\mathrm{n} / \mathrm{a}$ \\
L1-C02 & 209 & 204.0 & 9.7 & 1.02 & 1.21 & $\mathrm{n} / \mathrm{a}$ \\
L1-C03 & 204 & 204.0 & 15.5 & 1.00 & 1.21 & $\mathrm{n} / \mathrm{a}$ \\
L2-M02 & 215 & 204.0 & 8.1 & 1.05 & 1.21 & $\mathrm{n} / \mathrm{a}$ \\
L2-M04 & 216 & & & & & \\
L2-M05 & 229 & 204.0 & 7.6 & 1.06 & 1.21 & $\mathrm{n} / \mathrm{a}$ \\
\hline
\end{tabular}

Large-scale dowelled hold-downs (Ottenhaus et al. 2018c)

\begin{tabular}{lcccccc}
\hline L2-01M & 1310.9 & 854 & 6.3 & $\mathbf{1 . 5 4}$ & 1.45 & 1.67 \\
L2-02C & 1291.8 & 854 & 6.5 & $\mathbf{1 . 5 1}$ & 1.45 & 1.67 \\
L2-03C & 1238.8 & 854 & 6.0 & 1.45 & 1.45 & 1.67 \\
& & & & & & \\
L2-04C & 1268 & 854 & 6.3 & $\mathbf{1 . 4 9}$ & 1.45 & 1.67 \\
& & & & & & \\
L3-01M & 1285.6 & 747 & 7.1 & $\mathbf{1 . 7 2}$ & 1.58 & 1.85 \\
& & & & & & \\
L3-04C & 1177.3 & 747 & 6.0 & 1.58 & 1.58 & 1.85 \\
\hline
\end{tabular}


Table 5. Validation of $\gamma_{R d, t h}$ with data from Jorissen (1998).

\begin{tabular}{|c|c|c|c|c|c|c|c|}
\hline $\begin{array}{l}\text { series } \\
\text { name }\end{array}$ & $\begin{array}{l}t_{1} \\
{[\mathrm{~mm}]}\end{array}$ & $\begin{array}{l}t_{2} \\
{[\mathrm{~mm}]}\end{array}$ & $\begin{array}{l}F_{\text {max }, \exp } \\
{[\mathrm{kN}]}\end{array}$ & $\begin{array}{l}F_{u, k, p r e d} \\
{[\mathrm{kN}]}\end{array}$ & $\begin{array}{l}\mu \\
{[-]}\end{array}$ & $\begin{array}{l}\gamma_{R d, \exp } \\
{[-]}\end{array}$ & $\begin{array}{c}\gamma_{R d, t h} \\
{[-]}\end{array}$ \\
\hline D31107 & 24 & 48 & 14.22 & 10.43 & 6.00 & 1.36 & 1.42 \\
\hline D33137 & 24 & 48 & 35.29 & 31.29 & 4.69 & 1.13 & 1.42 \\
\hline D33157 & 24 & 48 & 34.26 & 31.29 & 5.59 & 1.09 & 1.42 \\
\hline D35137 & 24 & 48 & 60.82 & 52.16 & 4.38 & 1.17 & 1.42 \\
\hline D35157 & 24 & 48 & 60.44 & 52.16 & 4.45 & 1.16 & 1.42 \\
\hline D41107 & 36 & 48 & 12.10 & 11.87 & 6.67 & 1.02 & 1.44 \\
\hline D45157 & 36 & 48 & 62.10 & 59.36 & 5.22 & 1.05 & 1.44 \\
\hline D61107 & 48 & 48 & 16.06 & 13.83 & 4.00 & 1.16 & 1.45 \\
\hline D65157 & 48 & 48 & 72.34 & 69.14 & 4.12 & 1.05 & 1.45 \\
\hline D81107 & 59 & 72 & 24.03 & 15.46 & 5.00 & 1.55 & 1.41 \\
\hline D83137 & 59 & 72 & 49.88 & 46.39 & 4.55 & 1.08 & 1.41 \\
\hline D83157 & 59 & 72 & 50.49 & 46.39 & 4.17 & 1.09 & 1.41 \\
\hline D89157 & 59 & 72 & 186.32 & 139.17 & 4.56 & 1.34 & 1.41 \\
\hline
\end{tabular}


Table 6. Validation of $\gamma_{R d, t h}$ with data from Dorn et al. (2013).

\begin{tabular}{lllllll}
\hline $\begin{array}{l}\text { series } \\
\text { name }\end{array}$ & $t$ & $F_{\max , \exp }$ & $F_{u, k, \text { pred }}$ & $\mu$ & $\gamma_{R d, \exp }$ & $\gamma_{R d, \text { th }}$ \\
{$[\mathrm{mm}]$} & {$[\mathrm{kN}]$} & {$[\mathrm{kN}]$} & {$[-]$} & {$[-]$} & {$[-]$} \\
\hline 1 & 45 & 27.50 & 19.48 & $\mathrm{D}$ & 1.41 & 1.51 \\
2 & 45 & 22.00 & 19.48 & $\mathrm{D}$ & 1.13 & 1.51 \\
$08 \mathrm{a}$ & 45 & 30.00 & 19.48 & $\mathbf{D}$ & $\mathbf{1 . 5 4}$ & 1.51 \\
10 & 45 & 24.00 & 19.48 & $\mathrm{D}$ & 1.23 & 1.51 \\
$16 \mathrm{a}$ & 45 & 23.00 & 19.48 & HD & 1.18 & 1.51 \\
$16 \mathrm{c}$ & 45 & 20.00 & 19.48 & HD & 1.03 & 1.51 \\
\hline
\end{tabular}




\section{Figure Captions}

Fig. 1 Theoretical overstrength components (modified from Jorissen and Fragiacomo (2011)). Figure by Ottenhaus (2019).

Fig. 2 Experimentally derived overstrength. Figure by Ottenhaus (2019a).

Fig. 3 European Yield Model for steel-to-timber connection with a) thick steel side plate and b) internal steel plate.

Fig. 4. a) Overstrength components. Figure by Ottenhaus (2019b), b) overstrength from variability in material properties and sources. Figure by Ottenhaus (2019c), c) overstrength from conservatism in analytical models and contributing sources. Figure by Ottenhaus (2019d).

Fig. 5 Model overstrength $\gamma_{\text {mod,Meff }}$ from using the effective bending moment instead of plastic bending moment.

Fig. 6 Load displacement curves, predicted strength, $F_{u, k, p r e d}$, upper bound overstrength $\gamma_{R d, t h} F_{u, k \text { pred }}$, for a) nailed connections in CLT, b) nailed connections in LVL, c) dowelled small-scale connections in CLT, d) dowelled small-scale connections in LVL. 


\title{
Analytical derivation and experimental verification of overstrength factors of dowel-type timber connections for capacity design
}

\author{
Lisa-Mareike Ottenhaus ${ }^{1 *}$, Minghao Li $^{2}$, Tobias Smith ${ }^{3}$ \\ ${ }^{1}$ Lecturer, School of Civil Engineering, the University of Queensland, Australia, \\ l.ottenhaus@uq.edu.au; (corresponding author, formerly Ph.D. candidate at University \\ of Canterbury, New Zealand) \\ ${ }^{2}$ Senior Lecturer, Department of Civil and Natural Resources Engineering, University \\ of Canterbury, New Zealand,minghao.li@canterbury.ac.nz; \\ ${ }^{3}$ PTL | Structural Timber Consultants, PO Box 8415, Christchurch 8041, New Zealand. \\ t.smith@ptlnz.com;
}




\title{
Analytical derivation and experimental verification of overstrength factors of dowel-type timber connections for capacity design
}

\begin{abstract}
In timber structures, connections traditionally provide ductility and energy dissipation under seismic loading. Capacity design ensures that the global structural response is ductile by applying overstrength to the design demand of brittle elements. Overstrength is often derived experimentally which is costly and time consuming. This paper proposes an analytical method to estimate the overstrength of dowel-type timber connections based on inherent material properties, thereby reducing the need for experimental testing. The method is validated with data from previous experiments and literature for dowelled, bolted, and nailed timber connections, and provides reasonably accurate upper-bound overstrength estimates.
\end{abstract}

Keywords: overstrength; capacity design; timber; connection; seismic

\section{Introduction}

Capacity design was originally developed in California in the 1960s and was promoted in New Zealand by Tom Paulay for seismic design of reinforced concrete structures (Park and Paulay 1975, Fardis 2018). Nowadays, capacity design is widely accepted in seismic design of steel and concrete structures and has recently gained popularity in the design of timber structures, with suggestions to include it in Chapter 8 of Eurocode 8 for the seismic design of timber structures (Follesa et al. 2011, Loss et al. 2013, Follesa et al. 2018).

Capacity design of timber structures ensures that ductile behaviour can be achieved under seismic loading by detailing ductile connections as the weakest link along the load path, and protecting all brittle timber elements from the overstrength of the ductile connections (Moroder et al. 2014, Fardis 2018). In this context, overstrength of the 
ductile connections is defined as the difference between the design strength, $F_{d}$, and the $95^{\text {th }}$-percentile of the actual strength distribution, $F_{0.95}$ (Jorissen and Fragiacomo 2011, Follesa et al. 2018). To ensure a structural system can mobilise its ductile potential, $F_{B R, d} \geq \gamma_{R d} F_{u, d}$ needs to be satisfied, i.e. the design capacity of the brittle elements, $F_{B R, d}$, needs to be higher than the ultimate actual capacity of the ductile connection, $F_{u, d}$. This realisation leads to the definition of an overstrength factor, $\gamma_{R d}$, that defines the gap between the design and ultimate probable capacity of the ductile connection $\left(F_{0.95}\right)$, beyond which the brittle failure modes should be verified.

Overstrength in timber connections stems from a variety of sources and can be difficult to define accurately. It is often derived experimentally and only limited overstrength data of few timber connection types is currently available in literature.

A component based overstrength concept, adapted from Jorissen and Fragiacomo (2011), is shown for dowel-type timber connections in Figure 1 and Equation 1 and the method presented in this paper is based on this overstrength definition

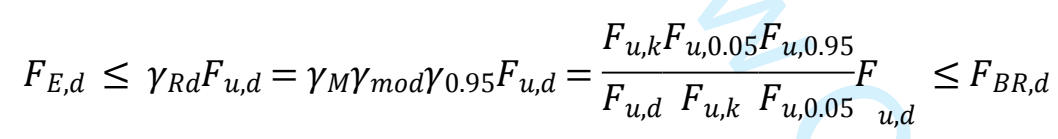

where $F_{E, d}$ is the design demand of the connection, $F_{u}$ is the ultimate ductile connection strength as defined in design codes with its characteristic and design values, $F_{u, k}$ and $F_{u, d}, \gamma_{M}$ is the partial material safety factor, $\gamma_{\bmod }$ accounts for conservatism of analytical models for design, and $\gamma_{0.95}$ quantifies the difference between the $5^{\text {th }}$ and $95^{\text {th }}$-percentile of the strength distribution ( $F_{0.05}$, and $F_{0.95}$, respectively).

Since $\gamma_{M}$ is defined in design codes, the only unknown components of the theoretical overstrength, defined as $\gamma_{R d, t h}$, are $\gamma_{\bmod }$ and $\gamma_{0.95}$. A method to estimate these two unknowns will be presented in this paper. While the connection design is based on the 
European yield model (EYM) in Eurocode 5 (2004), the overstrength concept holds for different material standards and material grades alike and reference to the respective material standards is made where appropriate.

It should be noted that there are more refined versions of this approach, such as the modified capacity design model by Trutalli et al. (2018). However, for the purpose of providing an analytical calculation method aimed at designers and practitioners, the method by Jorissen and Fragiacomo (2011) produces reasonably accurate results without introducing too much complexity, as will be presented in this paper.

\section{Overstrength theory in capacity design}

Experimental overstrength factors can be defined by $\gamma_{R d, \text { exp }}=F_{\max , \exp } / F_{u, k, p r e d}$ as shown in Figure 2, where $F_{\max , \exp }$ is the maximum capacity observed across a number of test specimens, and $F_{u, k, p r e d}$ is the predicted ultimate ductile capacity based on the EYM. This method has the drawback that the observed maximum capacity is generally smaller than the $95^{\text {th }}$-percentile of the connection strength distribution $\left(F_{\max , \exp } \leq F_{u, 0.95}\right)$ due to a limited sample size; thus non-conservative overstrength factors may sometimes be derived. Therefore, several researchers attempted to identify individual overstrength components and derived $\gamma_{R d, t h}$ for connections (Jorissen and Fragiacomo 2011, Schick at al. 2013, Brühl et al. 2014, Gavric et al. 2014, Vogt et al. 2014, Izzi et al. 2016, O'Ceallaigh and Harte 2019). An overview of some connection overstrength values in literature for connections in Glued Laminated Timber (Glulam) and Cross Laminated Timber (CLT) is given in Table 1 .

[Table 1 here]

Dowel-type connections with nails, screws, dowels and bolts are the most commonly used connection type in timber buildings. First introduced by Johansen (1949), the EYM is widely accepted as able to predict the ductile capacity of dowel-type 
connections with reasonable accuracy (Pedersen et al. 1999) and has been adopted by many timber design codes around the world including Eurocode 5 (2004). As an example, Figure 3 shows the possible failure modes defined in the EYM for a steel-totimber connection with a thick steel side plate or an internal steel plate. Equation 2 gives the respective prediction of the ultimate ductile capacity, $F_{u}$, per fastener and shear plane based on the timber thickness, $t$, fastener diameter, $d$, fastener bending moment, $M_{y}$, and timber embedment strength, $f_{h}$, as well as the rope effect, $F_{\text {rope }}$. For bolts and nails, the rope effect is determined as $F_{\text {rope }}=\min \left(F_{a x} / 4,0.25 F_{u}\right)$, where $F_{a x}$ is the axial capacity of the fastener, and is the $F_{u}$ Johansen part. $F_{\text {rope }}$ equals zero for smooth dowels.

$$
F_{u}=\min \left\{\begin{array}{c}
f_{h} t d\left[\sqrt{2+\frac{4 M_{h} t d}{f_{h} d t^{2}}-1}\right]+F_{\text {rope }} \\
2.3 \sqrt{M_{y} f_{h} d}+F_{\text {rope }}
\end{array}\right.
$$

\section{Analytical method to derive overstrength}

Figures $4 \mathrm{a}$ to $4 \mathrm{c}$ show a breakdown of the individual overstrength components that constitute $\gamma_{R d}$ based on the definitions in Equation 1. As the connection strength calculated by the EYM depends on the timber material embedment strength $f_{h}$ (which is correlated to timber density $\rho$ and fastener diameter) and the fastener bending moment $M_{y}$ (which is correlated to fastener yield strength $f_{y}$ and cross section), each overstrength component can be subdivided into a contribution from the timber and steel material, as shown in Figure $4 b$ and $4 c$.

In the following sections a detailed procedure is presented to analytically derive and quantify $\gamma_{\text {mod }}$ and $\gamma_{0.95}$ for dowel-type connections in the context of Eurocode 5 based on 
material properties. In addition, accidental overstrength, $\gamma_{\text {err }}$, is discussed which is introduced when materials with higher than specified grades are supplied.

\section{Material safety factor $\gamma_{M}$}

The material safety factor is defined as $\gamma_{M}=F_{u, k} / F_{u, d}$ and is given in the respective design standards: For dowelled connections in sawn timber, Eurocode 5 (2004) recommends $\gamma_{M}=1.3$, and NZS3603 (1993) recommends a strength reduction factor of $\phi=0.8$ which leads to $\gamma_{M}=1 / \phi=1.25$. In seismic design, however, $\gamma_{M}$ is not considered: NZS3603 (1993) recommends $\phi=1.0$ and Eurocode 8 (2004) recommends $\gamma_{M}=1.0$. Therefore, the total theoretical overstrength is reduced to $\gamma_{R d, t h}=\gamma_{\text {mod }} \gamma_{0.95}$

\section{Material variability within speficied grade $\gamma_{0.95}$}

Jorissen and Fragiacomo (2011) attributed $\gamma_{0.95}=F_{u, 0.95} / F_{u, 0.05}$ to the scatter of the connection strength properties. The $5^{\text {th }}$ and $95^{\text {th }}$-percentiles of the connection strength distribution are directly related to the variability of the timber material and steel fasteners within the specified material grade. It is thus possible to estimate $\gamma_{0.95}$ by inserting the $5^{\text {th }}$ and $95^{\text {th }}$-percentile of the timber density and steel yield strength distributions into the EYM. To determine $\gamma_{0.95}$, either upper and lower bounds of the material grade can be used, although supplier information is preferable as it yields less conservative $\gamma_{0.95}$ values. Timber suppliers generally record density as well as other material properties to determine the material grade. In a similar manner, steel fastener manufacturers conduct testing for each batch and steel grade produced. The method to determine $\gamma_{0.95}$ is shown for the calculation of $F_{u}$ of dowel-type timber connections with parallel-to-grain loading in Equations 3.1 through 3.3, where the embedment strength formulas in Equation 3.2 are given in Eurocode 5 for sawn timber, the CLT Handbook for CLT (FPInnovations 2011), and by Franke and Quenneville (2011) for LVL. The 
resulting overstrength factors then depend on the governing EYM failure mode, which in turn depends on the timber thickness, $t$, fastener diameter, $d$, and material properties. Upper-bound overstrength subcomponents from the timber and steel contributions, $\gamma_{0.95, f h}$ and $\gamma_{0.95, M y}$, are given in Table 2.

$$
\begin{gathered}
\gamma_{0.95}=\gamma_{0.95, f h} \gamma_{0.95, M y} \\
\gamma_{0.95 . f h}=\frac{F\left(f_{h, 0.95}\right)}{F\left(f_{h, 0.05}\right)} \text { where } f_{h, 0.05}=f\left(\rho_{0.05}\right) ; f_{h, 0.95}=f\left(\rho_{0.95}\right) \\
\text { and } f_{h}=\left\{\begin{array}{cc}
0.082(1-0.01 d) \rho & \text { for sawn timber } \\
0.031(1-0.015 d) \rho^{1.16} & \text { for } C L T \\
0.075(1-0.0037 d) \rho & \text { for } L V L
\end{array}\right. \\
\gamma_{0.95 . M y}=\frac{F\left(M_{y, 0.95}\right)}{F\left(M_{y, 0.05}\right)} \text { where } M_{y, 0.05}=f_{y, 0.05} d^{3} / 6 \text { and } M_{y, 0.95}=f_{y, 0.95} d^{3} / 6
\end{gathered}
$$

\section{Conservatism in Models $\gamma_{\bmod }$}

Analytical model overstrength, $\gamma_{\text {mod }}$, quantifies the difference between the $5^{\text {th }}$-percentile of the actual connection strength, $F_{u, 0.05}$, and the characteristic strength, $F_{u, k}$, calculated by the EYM. As shown in Figure 4c, $\gamma_{\text {mod }}$ stems from conservatism in the material models as well as the difference between the characteristic yield strength and nominal yield strength of steel fasteners which is prescribed by design codes. The overstrength in the material models encompasses the conservatism of semi-empirical calculation formulas as well as the approximation error of the analytical formulas used to predict the connection strength (Jorissen and Fragiacomo 2011).

Eurocode 5 (2004) uses the effective bending yield moment $M_{e f f}=0.3 d^{2.6} f_{u}$ to calculate the fastener yield moment, $M_{y}$, based on the assumption that full plasticisation of the cross sections of stocky fasteners is hardly achieved in timber connections (Blaß et al. 2001). However, as Stehn and Johnsson (2002) pointed out, this is due to timber splitting which leads to mode cross-over from a ductile response to brittle failure of the 
joint itself. A detailed explanation of mode cross-over due to reduced brittle resistance is given in Stehn and Johnsson (2002), Novis et al. (2016), and Ottenhaus et al. (2018a). In highly ductile connections, fasteners can achieve full plastic hinges with $M_{y}=M_{p}=f_{y}$ $d^{3} / 6$. If capacity design is used to achieve ductile connection behaviour, the use of $M_{y}=M_{\text {eff }}$ instead of $M_{y}=M_{p}$ can introduce significant model overstrength (Schick et al. 2016), which, depending on the governing EYM mode, can reach up to $\gamma_{\text {mod,Meff }}=1.44$, as shown in Figure 5 for the allowable steel strength range of AS/NZS 4671 (2001). Semi-empirical material models for timber connection design are usually established by conservatively calibrating an analytical model to experimental data. Examples of such models are the different embedment strength formulas given in Equation 3.2. If sufficient data is gathered the difference between calculated and experimental embedment strength can be minimised, which may lead to small overstrength factors $\gamma_{m o d, f h}$

The difference between the $5^{\text {th }}$-percentile of the steel yield strength, $f_{y, 0.05}$, and nominal strength, $f_{y, n o m}$, introduces overstrength as well. Karmazínová and Melcher (2012) conducted experiments on grade S235 and S355 steel (grades according to Eurocode 3) and another set of tensile test data of Grade 300 and Grade 500 steel was made available by a New Zealand steel supplier (grades according to AS/NZS 4671 2001).

Overstrength from nominal steel strength, $\gamma_{\text {mod,nom }}$, can be estimated by inserting $f_{y, 0.05}$ and $f_{y, n o m}$ into the EYM calculations. The resulting upper-bound overstrength values are given in Table 2 .

Finally, it should be noted that larger sample sizes result in smaller overstrength factors $\gamma_{0.95}$ and $\gamma_{\text {mod }}$ and that stress grading reduces the variability in timber material properties and therefore can reduce $\gamma_{0.95}$, as shown in Table 2 .

[Table 2 here] 


\section{Accidental overstrength $\gamma_{\mathrm{err}}$}

Accidental overstrength, $\gamma_{e r r}$, is introduced if the supplied material grade is significantly higher (stronger) than the specified grade due to the common misconception that a stronger material is 'better' (Misconel et al. 2016, Sandhaas et al. 2017). In practice, this mistake often goes unnoticed, since high strength steel is visually no different from mild steel and the error would only be discovered through experimental testing. Installation of the higher strength material can lead to unsafe seismic design since the connection may now no longer be the weakest link the in the strength hierarchy. Accordingly, the accidental overstrength can then be defined as $\gamma_{\text {err }}=F_{\text {supplied }} / F_{\text {specified }}$.

\section{Results of model validation and discussion}

The analytical method is checked against the results of dowelled and nailed hold-down connection tests conducted at structural engineering laboratories of the University of Canterbury. These hold-down connections were designed as highly ductile elements for mass timber shear walls that could develop overstrength and ductile behaviour and thereby qualified as potential ductile elements following the capacity design approach. As suggested by Smith et al. 2006, highly ductile behaviour is defined as $\mu=\Delta_{u} / \Delta_{y}>6$, where $\mu$ is the connection ductility, $\Delta_{u}$ is the ultimate displacement, and $\Delta_{y}$ is the yield displacement. Furthermore, the analytical method is further checked against previous experimental datasets from literature. For a detailed step-by-step procedure and example calculations refer to Ottenhaus et al. $2018 \mathrm{~d}$.

\section{Validation with experimental data from previous testing}

Table 3 gives the material input properties for the characteristic strength prediction, $F_{u, k, p r e d}$, of the connection tests, as well as theoretical overstrength prediction, $\gamma_{R d, t h}$. Various connection layouts with expected ductile behaviour were subjected to 
monotonic and cyclic loading (ISO and CUREE loading protocols for nailed connections and ISO loading for dowelled connections as described in Ottenhaus et al. 2016).

The nailed hold-down connections consisted of fifteen ø4x100mm nails and $10 \mathrm{~mm}$ thick steel side plates. The nails were spaced densely in LVL ( $4 d$ fastener spacing parallel to grain and $3 d$ perpendicular to grain) and widely in CLT ( $8 d$ fastener spacing parallel to grain and $6 d$ perpendicular to grain). Nail bending tests were conducted to determine the nail yield moment from which the yield strength was derived as $f_{y}=$ $530 \mathrm{MPa}$. More information on the nailed hold-down connection tests can be found in Ottenhaus et al. 2016.

The dowelled hold-down connections consisted of an internal steel plate and ø20 mm dowels with specified Grade 300 steel. The small-scale tests were conducted in CLT and LVL with varying fastener spacing and the large-scale test were conducted in CLT and a CLT-LVL layup. More information regarding the dowelled connection test setups can be found in Ottenhaus et al. 2018a and 2018c.

It was found that EYM mode iii in Equation 2 was governing for all nailed hold-downs and mode ii was the governing case for all dowelled hold-downs.

Overstrength from the material strength distribution within the designated grade, $\gamma_{0.95}=$ $F_{0.95} / F_{0.05}$ was calculated using the EYM and Equations 3.2 and 3.3. and data from Table 2.

Model overstrength, $\gamma_{\text {mod }}=F_{0.05} / F_{k}$ was estimated using the EYM by inserting $5^{\text {th }}$ percentile timber density, $\rho_{0.05, \exp }$, into embedment strength formulas (Equation 3.2), and by contrasting the results with experimental data from Ottenhaus and $\mathrm{Li}$ (2018). In addition, nominal fastener yield strength was compared to $5^{\text {th }}$-percentile yield strength. 
It was found that $\gamma_{\text {mod }}$ is relatively low (around 10\%). Note that for the nailed connections, no embedment tests were conducted and $\gamma_{\text {mod }}$ was estimated based on the results for dowelled small-scale connections.

For the large-scale connection tests, Grade 500 dowels instead of Grade 300 dowels were supplied which was discovered during tensile and three-point bending tests (Ottenhaus et al. 2018b), which means the overstrength estimates were nonconservative. The error can be expressed as accidental overstrength $\gamma_{\text {err }}=F\left(f_{y}=500 \mathrm{MPa}\right)$ / $F\left(f_{y}=300 \mathrm{MPa}\right)$. Note that accidental overstrength cannot be predicted or accounted for in practice unless experimental testing is carried out. In consequence, additional overstrength due to supply of a stronger steel grade can jeopardise seismic safety and cause premature brittle failure since the ductile connection response may now no longer be the weakest link in the strength hierarchy. Therefore, it is important raise awareness of this issue in the professional community and to educate suppliers on capacity design.

A summary of the resulting theoretical overstrength and its components is given in Table 3. In those cases where no experimental data was available, a conservative assumption was made.

[Table 3 here]

Table 4 gives the various connection tests results as well as ductility, $\mu$, experimental overstrength, $\gamma_{R d, \exp }=F_{\text {max,exp }} / F_{u, k, \text { pred }}$, and theoretical overstrength, $\gamma_{R d, t h}=\gamma_{0.95} \gamma_{\text {mod }}$. The cases where the overstrength estimation was non-conservative $\left(\gamma_{R d, \exp }>\gamma_{R d, t h}\right)$ are printed in bold. For the small-scale dowelled connections tests this was due to a specimen that exhibited exceptional strength. For the large-scale dowelled connection tests this was due to accidental overstrength and when $\gamma_{\text {err }}$ was considered, the 
overstrength prediction was conservative. Note that the estimated overstrength values for dowels are smaller than the previously proposed overstrength factor of 1.68 (Ottenhaus et al. 2017), which was conservatively based on upper-bound overstrength component values that did not take the governing EYM mode into account.

[Table 4 here]

Figure 6 visualises some of the data presented in Table 4 and displays the load displacement and backbone curves as well as predicted strengths for the small-scale nailed and dowelled hold-down connection tests. As can be seen from Table 4 and Figure 6, the overstrength estimation yielded conservative results in most cases and generally agreed well with the experimental data $\left(\max \gamma_{R d, \exp } \approx \gamma_{R d, t h}\right.$, and $\gamma_{R d, t h}$ was on average $18 \%$ conservative and non-conservative in one case), except when the wrong steel grade was supplied.

\section{Validation with experimental data from literature}

The previously described method was validated with experimental data from literature. The studies were selected based on the following criteria:

- failure was truly ductile (i.e. $\mu>4$ )

- data regarding density distribution and steel material grade was either included in the publication or made available

- connection geometry was described sufficiently well to estimate capacity

- load displacement curves were made available to estimate ductility $\mu$

Jorissen (1998) conducted experiments on symmetric bolted timber-to-timber connections in sawn European Spruce. The slenderness ratio of the connection specimens was varied by changing the timber member thicknesses of the outer 
members, $t_{1}$, and inner member, $t_{2}$. The average timber density and standard deviation are given in Jorissen's thesis as $\rho_{a v}=450 \mathrm{~kg} / \mathrm{m}^{3}$ and $\sigma=44 \mathrm{~kg} / \mathrm{m}^{3}$, respectively. The bolts were specified M12 $(d=12 \mathrm{~mm})$ grade 4.6 with a nominal yield strength of $f_{y, n o m}=$ $240 \mathrm{MPa}$ (DIN931 1987). An upper-bound for the yield strength was estimated as $f_{y, 0.95}$ $=300 \mathrm{MPa}$ based on the allowable range given for mild steel in AS/NZS 4671 (2001) as this is the only commonly used material standard that specifies an upper-bound strength for mild steel. Table 5 gives the experimental strength, predicted strength, experimental overstrength, ductility, as well as predicted overstrength for those test series that responded in a ductile $(\mu>4)$ or highly ductile $(\mu>6)$ manner and produced overstrength, with a conservative assumption of $\gamma_{\text {mod }}=1.15$. It should be noted that the predicted strength values in Table 5 may differ from those in Jorisson 1998 since the plastic yield moment was used for the strength prediction in this paper. The cases where the overstrength estimation was non-conservative $\left(\gamma_{R d, \exp }>\gamma_{R d, t h}\right)$ are printed in bold. [Table 5 here]

Dorn et al. (2013) conducted experiments on single dowel connections in double shear with an internal steel plate. The dowel diameter was $d=12 \mathrm{~mm}$ with varying timber thickness, $t$. According to Dorn et al., the widths of the specimens were chosen in order to produce one of the three main ductile responses in Eurocode 5 (shown in Figure 3b) and it was found that the ductility increases with the width of the specimen. The timber species was Norwegian Spruce and the density percentiles were based on the values given in the publication with assumed grade $\mathrm{C} 24: \rho_{0.05}=350 \mathrm{~kg} / \mathrm{m}^{3}$ and $\rho_{0.95}=510$ $\mathrm{kg} / \mathrm{m}^{3}$. In the publication only a value for $f_{u}=708 \mathrm{MPa}$ was given and hence an 'educated guess' had to be made regarding the nominal steel strength and $95^{\text {th }}$-percentile strength: $f_{y, \text { nom }}=500 \mathrm{MPa}$ and $f_{y, 0.95}=625 \mathrm{MPa}$, based on the allowable ranges for $f_{y}$ as well as $f_{u} / f_{y}$ ratios given in other material standards. The maximum experimental 
strength values were derived from the graphs provided in the publication. The ductility was estimated based on the graphs as ductile $(\mathrm{D}, \mu>4)$ or highly ductile (HD, $\mu>6)$. The results are given in Table 6 and those cases where the predicted overstrength was non-conservative are printed in bold.

[Table 6 here]

As can be seen from Tables 8 and 9, the overstrength factors derived by the analytical method generally agreed well with the experimental data $\left(\max \gamma_{R d, \exp } \approx \gamma_{R d, t h}\right.$, and $\gamma_{R d, t h}$ was on average $23 \%$ conservative and non-conservative in two cases).

\section{Conclusions}

This paper presented an analytical method to derive overstrength factors of dowel-type timber connections that are commonly designed as potential ductile elements in timber structures following the capacity design approach. The analytical derivation is based on ductile failure modes in the European yield model considering the variability of material properties and conservatism in semi-empirical embedment and dowel yield moment models. The material properties used in the analytical method are either specified by strength classes in design codes or obtained from steel and timber suppliers.

Experimental data of nailed and dowelled hold-down connections that are designed as ductile elements in mass timber shear walls as well as additional connection test data in literature were used to check the accuracy of the analytical method. It was found that the analytical overstrength factors generally agreed well with the test results and were slightly conservative in most cases, except when the supplied steel yield strength well exceeded that of the specified steel grade. 
In general, this method provides reasonably accurate estimations of the upper-bound overstrength for dowel-type connections made of bolts, dowels, and nails, so that it has the potential to replace costly experimental derivation of connection overstrength. Provided accurate material models such as the ones presented in this paper are used for embedment strength and fastener strength calculations (i.e. use plastic fastener bending moment $M_{p}$ rather than effective yield moment $M_{e f f}$, the only required input data is the timber and steel material grade. However, where possible supplier information should be used to reduce conservatism in the overstrength estimation to yield a more economic design. This method therefore can provide designers with a relatively simple tool to calculate overstrength factors for specific ductile connection layouts without making overly conservative assumptions in capacity design.

It should also be noted that while the presented method is independent of material, fastener type, and connection layout, a good understanding of conservatism in the underlying analytical models is necessary to apply the method to other fastener types such as screws and rivets. In future research, the methodology may thus be extended to and validated for different fasteners types, connection layouts, loading scenarios, and timber materials. 


\section{Word count main text 3644}

Word count abstract 100

\section{Acknowledgements}

The research was funded jointly by Natural Hazards Research Platform, Earthquake Commission (EQC) of New Zealand, and ARC Research Hub for Advanced Solutions to Transform Tall Timber Buildings in Australia. The material was provided by XLam NZ Ltd and Nelson Pine Industries Ltd and their support is gratefully acknowledged.

\section{References}

AS/NSZ (Standard Australia, Standards New Zealand) (2005). "Structural Laminated Veneer Lumber." AS/NZS 4357.0:2005, Sydney, Wellington.

AS/NSZ (Standard Australia, Standards New Zealand) (2001). "Steel reinforcing materials." AS/NZS 4671:2001, Sydney, Wellington.

Blaß, H. J., Bienhaus, A., and Krämer, V. (2001). "Effective bending capacity of dowel-type fasteners." Proceedings of the International RILEM Symposium Joints in Timber Structures, Stuttgart, 22, 71-88.

Brühl, F., Schänzlin, J., and Kuhlmann, U. (2014). "Ductility in Timber Structures: Investigations on Over-Strength Factors." RILEM Bookseries (Vol. 9) Springer Netherlands, Dordrecht.

DIN (Deutsches Institut fur Normung ) (1987). "Sechskantschauben mit Schaft, Gewine M 1.6 bis M39, Produktklassen A und B.” DIN931 Teil 1, Berlin.

Dorn, M., de Borst, K., and Eberhardsteiner, J. (2013). "Experiments on dowel-type timber connections." Engineering Structures, 47, 67-80.

CEN (European Committee for Standardization) (2005). "Design of steel structures, Part 1.1." Eurocode 3, Brussels

CEN (European Committee for Standardization) (2004). "Design of timber structures, Part 1.1." Eurocode 5, Brussels

CEN (European Committee for Standardization) (2004). "Design of structures for earthquake resistance, Part 1." Eurocode 8, Brussels

CEN (European Committee for Standardization) (2016). "Structural timber. Strength classes." EN338:2016, Brussels. 
Fardis, M. N. (2018). Capacity design: Early history. Earthquake Engineering and Structural Dynamics, 47(14), 2887-2896.

Follesa, M., Fragiacomo, M., \& Lauriola, M. (2011). A proposal for revision of the current timber part (Section 8) of Eurocode 8 Part. Proceedings of CIB-W18 meeting forty-four, Alghero, Italy, 2011.

Follesa, M., Fragiacomo, M., Casagrande, D., Tomasi, R., Piazza, M., Vassallo, D., ... Rossi, S. (2018). The new provisions for the seismic design of timber buildings in Europe. Engineering Structures, 168(December 2017), 736-747.

FPInnovations (2011). CLT Handbook. E. Karacabeyli and R. Desjardins, Eds.

Fragiacomo, M., Dujic, B., and Sustersic, I. (2011). "Elastic and ductile design of multi-storey crosslam massive wooden buildings under seismic actions." Engineering Structures, 33(11), 3043-3053.

Franke, S., and Quenneville, P. (2011). "Bolted and dowelled connections in radiate pine and laminated veneer lumber using the European yield model." Australian Journal of Structural Engineering, 12(1), 13-28.

Gavric, I., Fragiacomo, M., and Ceccotti, A. (2014). "Cyclic behaviour of typical metal connectors for cross-laminated (CLT) structures." Materials and Structures, 48(6), 1841-185.

Gavric, I., Fragiacomo, M., and Ceccotti, A. (2015). "Cyclic behavior of typical screwed connections for cross-laminated (CLT) structures." European Journal of Wood and Wood Products, 73(2), 179-191.

Izzi, M., Flatscher, G., Fragiacomo, M., \& Schickhofer, G. (2016). “Experimental investigations and design provisions of steel-to-timber joints with annular-ringed shank nails for CrossLaminated Timber structures." Construction \& building materials, 122, 446-457.

Johansen, K. W. (1949). "Theory of Timber Connections." Int Assoc Bridge and Struct Eng, 9, $249-262$.

Jorissen, A. (1998). "Double shear timber connections with dowel type fasteners." PhD thesis, TU Delft.

Jorissen, A., and Fragiacomo, M. (2011). "General notes on ductility in timber structures." Engineering Structures, 33(11), 2987-2997.

Karmazínová, M., and Melcher, J. (2012). "Influence of Steel Yield Strength Value on Structural Reliability of Steel Yield Strength." Recent Researches in Environmental and Geological Sciences, 2012, 441-446.

Loss, C., Zonta, D., \& Piazza, M. (2013). On Estimating the Seismic Displacement Capacity of Timber Portal-Frames. Journal of Earthquake Engineering, 17(6), 879-901. 
Misconel, A., and Ballerini, M. (2016). "Steel-To-Timber Joints of Beech-LVL With Very High Strength Steel Dowels". Proceedings of World Conference on Timber Engineering (WCTE2016), Vienna.

Moroder, D., Smith, T., Pampanin, S., Palermo, A., and Buchanan, A. H. (2014). "Design of Floor Diaphragms in Multi-Storey Timber Buildings". Proceedings of International Network on Timber Engineering Research Meeting, 2014, Bath.

Novis, S. A. L., Jacks, J., \& Quenneville, P. (2016). Predicting the Resistance and Displacement of Timber Bolted Connections. Proceedings of World Conference on Timber Engineering (WCTE2016), Vienna.

NZS (Standards New Zealand). (1993). “Timber Structures Standard.” NZS3603:1993, Wellington.

O'Ceallaigh, C., \& Harte, A. M. (2019). "The elastic and ductile behaviour of CLT wall-floor connections and the influence of fastener length." Engineering Structures, 189(March), 319331 .

Ottenhaus, L.-M. (2019). "theoretical overstrength.” Figshare (Figure). doi: $10.6084 / \mathrm{m} 9$. figshare.7834736v3

Ottenhaus, L.-M. (2019a). “experimental overstrength.” Figshare (Figure). doi: $10.6084 / \mathrm{m} 9$. figshare.7834751v2

Ottenhaus, L.-M. (2019b). "Overstrength Components.” Figshare (Figure). doi: 10.6084/m9.figshare.8239892.v1

Ottenhaus, L.-M. (2019a). “Overstrength from material variability.” Figshare (Figure). doi: 10.6084/m9.figshare.8239898.v1

Ottenhaus, L.-M. (2019d). “Overstrength from model conservatism.” Figshare (Figure). doi: 10.6084/m9.figshare.8239901.v2

Ottenhaus, L.-M., Li, M., Smith, T., \& Quenneville, P. (2016). Ductility of dowelled and nailed CLT and LVL connections under monotonic and cyclic loading. Australian Earthquake Engineering Society 2016 Conference.

Ottenhaus, L.-M., and Li, M. (2018). "Embedment Strength of New Zealand Cross Laminated Timber." New Zealand Timber Design Journal, 26(1), 12-16.

Ottenhaus, L.-M., Li, M., Smith, T., and Quenneville, P. (2018a). "Mode Cross-Over and Ductility of Dowelled LVL and CLT Connections under Monotonic and Cyclic Loading." Journal of Structural Engineering, 144(7), 1-10.

Ottenhaus, L.-M., Li, M., and Smith, T. (2018b). "Overstrength of large-scale dowelled connections in CLT." Proceedings of NZSEE Conference, 2018, Auckland. 
Ottenhaus, L.-M., Li, M., and Smith, T. (2018c). "Structural performance of large-scale dowelled CLT connections under monotonic and cyclic loading." Engineering Structures, 176(August), 41-48.

Ottenhaus, L.-M., Li, M., and Smith, T. (2018d). "Analytical Method to Derive Overstrength of Dowel-Type Connections." Proceedings of International Network on Timber Engineering Research Meeting, 2018, Tallinn.

Ottenhaus, L.-M., Li, M., Smith, T., and Quenneville, P. (2017). "Overstrength of dowelled CLT connections under monotonic and cyclic loading." Bulletin of Earthquake Engineering.

Park, R., and Paulay, T. (1975). "Reinforced Concrete Structures." Hoboken, NJ, USA: John Wiley \& Sons.

Pedersen, M. U., Clorius, C. O., Damklide, L., Hoffmeyer, P., and Eskildsen, L. (1999). "Dowel type connections with slotted-in steel plates." Proceedings of CIB-W18 meeting thirty-two, Graz, Austria, 1999.

Popovski, M., Prion, H. G., and Karacabeyli, E. (2002). "Seismic performance of connections in heavy timber construction." Canadian Journal of Civil Engineering, 29(3), 389-399.

Sandhaas, C., and van de Kuilen, J. W. G. (2017). "Strength and stiffness of timber joints with very high strength steel dowels." Engineering Structures, 131, 394-404.

Schick, M., Seim, W., and Kruger, S. (2016). "Dowel-Type Fasteners with Small Diameters Review and Improvement of Design Formulations." Proceedings of World Conference on Timber Engineering (WCTE2016), Vienna.

Schick, M., Vogt, T., and Seim, W. (2013). "Connections and anchoring for wall and slab elements in seismic design." Proceedings of CIB-W18 meeting, Vancouver, Canada, 2013, 31332 .

Smith, I., Asiz, A., Snow, M., \& Chui, Y. H. (2006). Possible Canadian / ISO Approach to Deriving Design Values From Test Data. Proceedings of CIB-W18 meeting thirty-nine

Stehn, L., and Johnsson, H. (2002). "Ductility Aspects in Nailed Glue Laminated Timber Connection Design.” Journal of Structural Engineering, 128(3).

Trutalli, D., Marchi, L., Scotta, R., \& Pozza, L. (2018). "Capacity design of traditional and innovative ductile connections for earthquake-resistant CLT structures." Bulletin of Earthquake Engineering

Vogt, T., and Seim, W. (2014). "Advanced modelling of timber-framed wall elements for application in engineering practice." Proceedings of International Network on Timber Engineering Research Meeting, Bath, 2014. 


\section{Notations}

The following symbols are used in this paper:

\begin{tabular}{|c|c|}
\hline$d$ & fastener diameter $\varnothing$ \\
\hline$F_{0.05}$ & $5^{\text {th }}$-percentile of the strength distribution \\
\hline$F_{0.95}$ & $95^{\text {th }}$-percentile of the strength distribution \\
\hline$F_{a x}$ & axial strength used to determine the rope effect $F_{\text {rope }}$ \\
\hline$F_{B R}$ & brittle strength \\
\hline$f_{\text {calc }}$ & calculated strength \\
\hline$F_{d}$ & design strength \\
\hline$F_{E, d}$ & design demand \\
\hline$F_{\text {exp }}$ & experimentally measured strength \\
\hline$f_{h}$ & timber embedment strength \\
\hline$F_{k}$ & characteristic strength \\
\hline$F_{\max }$ & maximum strength \\
\hline$f_{\text {nom }}$ & nominal strength \\
\hline$F_{\text {pred }}$ & predicted strength \\
\hline$F_{\text {rope }}$ & rope effect \\
\hline$F_{\text {specified }}$ & specified strength \\
\hline$F_{\text {supplied }}$ & supplied strength \\
\hline$F_{u}$ & ultimate ductile strength \\
\hline$f_{u}$ & fastener ultimate tensile strength \\
\hline$f_{y}$ & fastener yield strength \\
\hline$f_{y, \text { nom }}$ & nominal fastener yield strength \\
\hline$M_{y}$ & fastener bending moment \\
\hline$M_{e f f}$ & effective fastener bending moment \\
\hline$M_{p}$ & plastic fastener bending moment \\
\hline$t$ & timber member thickness \\
\hline$\gamma_{0.95}$ & overstrength factor to account for material strength distribution \\
\hline$\gamma_{0.95, f h}$ & overstrength from embedment strength distribution \\
\hline$\gamma_{0.95, M y}$ & overstrength from fastener yield strength distribution \\
\hline$\gamma_{\text {err }}$ & accidental overstrength $\gamma_{\text {err }}=F_{\text {supplied }} / F_{\text {specified }}$ \\
\hline$\gamma_{M}$ & partial material safety factor \\
\hline$\gamma_{\text {mod }}$ & overstrength factor to account for conservatism of analytical models \\
\hline$\gamma_{\text {mod,fh }}$ & overstrength from conservatism in embedment strength models \\
\hline$\gamma_{\text {mod,Meff }}$ & overstrength from using $M_{e f f}$ rather than $M_{p}$ \\
\hline$\gamma_{\text {mod,nom }}$ & overstrength from nominal fastener yield strength \\
\hline$\gamma_{R d}$ & overstrength factor \\
\hline$\gamma_{R d, \exp }$ & experimentally established overstrength factor $\gamma_{R d, \text { exp }}=F_{\max , \exp } / F_{u, k, p r e d}$ \\
\hline$\gamma_{R d, t h}$ & theoretical connection overstrength factor $\gamma_{R d, t h}=\gamma_{\bmod } \gamma_{0.95}$ \\
\hline$\Delta_{u}$ & ultimate displacement \\
\hline$\Delta y$ & yield displacement \\
\hline$\mu$ & ductility $\mu=\Delta_{u} / \Delta_{y}$ \\
\hline$\rho$ & timber density \\
\hline$\rho_{a v}$ & average timber density \\
\hline
\end{tabular}


$\sigma \quad$ standard deviation

$\phi \quad$ strength reduction factor 


\section{Tables}

Table1. Overstrength values in literature for different timber connections.

\begin{tabular}{|c|c|c|c|c|c|}
\hline Author & Connection type & $\gamma_{\text {mod }}$ & $\gamma_{0.95}$ & $\gamma_{R d, t h}$ & $\gamma_{R d, \exp }$ \\
\hline NZS3603 (1993) & nailed connections, sawn & & & 1.6 & \\
\hline Popovski et al. (2002) & bolted connections, Glulam & & & & $1.5-2.0$ \\
\hline $\begin{array}{l}\text { Fragiacomo et al. } \\
\text { (2011) }\end{array}$ & nailed hold-downs, CLT & & & & 1.3 \\
\hline Schick et al. (2013) & nailed hold-downs, CLT & $1.0-1.8$ & $1.0-1.4$ & $1.1-2.5$ & \\
\hline Gavric et al. (2014) & nailed hold-downs, CLT & $1.3-2.8$ & $1.2-1.4$ & $1.5-4.0$ & \\
\hline Vogt et al. (2014) & nailed hold-downs, CLT & 1.3 & 1.3 & 1.7 & \\
\hline Gavric et al. (2015) & screwed connections, CLT & & & & 1.6 \\
\hline \multirow[t]{4}{*}{ Izzi et al. (2016) } & annular-ringed shank nails, & & & & \\
\hline & CLT, withdrawal & 1.13 & 1.76 & 2.0 & 1.8 \\
\hline & lateral load parallel to grain & 1.44 & 1.27 & 1.8 & 1.3 \\
\hline & lateral load perp. to grain & 1.48 & 1.53 & 2.3 & 1.5 \\
\hline O'Ceallaigh and Harte & screwed brackets, CLT (C16) & & & & \\
\hline \multirow[t]{2}{*}{ (2019) } & tension & $1.1-1.4$ & $1.5-1.9$ & 2.5 & \\
\hline & shear & $1.1-1.3$ & $1.2-1.5$ & 2.1 & \\
\hline
\end{tabular}


Table 2. Upper-bound embedment-related material overstrength $\gamma_{0.95, f h}$ from timber density distribution, upper-bound fastener-related material overstrength $\gamma_{0.95, M y}$ from steel yield strength distribution and allowable range, and upper-bound model overstrength $\gamma_{\text {mod,nom }}$ from nominal steel strength.

\begin{tabular}{|c|c|c|c|c|}
\hline & sample size & $\rho_{0.05}\left[\mathrm{~kg} / \mathrm{m}^{3}\right]$ & $\rho_{0.95}\left[\mathrm{~kg} / \mathrm{m}^{3}\right]$ & $\gamma_{0.95, f h}{ }^{\mathrm{d})}$ \\
\hline ungraded Radiata pine a) & $\mathrm{n} / \mathrm{a}$ & 402 & 608 & 1.51 \\
\hline LVL11 b) & 57 & 480 & 564 & 1.18 \\
\hline LVL13 b) & 67 & 500 & 585 & 1.17 \\
\hline $\mathrm{C} 18^{\mathrm{c})}$ & $\mathrm{n} / \mathrm{a}$ & 320 & 440 & 1.38 \\
\hline \multirow[t]{2}{*}{$\mathrm{C} 24^{\mathrm{c})}$} & $\mathrm{n} / \mathrm{a}$ & 350 & 490 & 1.40 \\
\hline & sample size & $f_{y, 0.05}\left[\mathrm{~kg} / \mathrm{m}^{3}\right]$ & $f_{y, 0.95}\left[\mathrm{~kg} / \mathrm{m}^{3}\right]$ & $\gamma_{0.95, M y}{ }^{\mathrm{d})}$ \\
\hline $\mathrm{S} 235^{\mathrm{e})}$ & 26 & 284 & 377 & 1.15 \\
\hline $\mathrm{S} 355^{\mathrm{e})}$ & 19 & 410 & 498 & 1.10 \\
\hline Grade $300^{\text {f) }}$ & $\mathrm{n} / \mathrm{a}$ & 300 & 380 & 1.13 \\
\hline \multirow[t]{2}{*}{ Grade 500 f) } & $\mathrm{n} / \mathrm{a}$ & 500 & 600 & 1.10 \\
\hline & sample size & $f_{y, \text { nom }}\left[\mathrm{kg} / \mathrm{m}^{3}\right]$ & $f_{y, 0.05}\left[\mathrm{~kg} / \mathrm{m}^{3}\right]$ & $\gamma_{\text {mod,nom }}{ }^{\mathrm{d})}$ \\
\hline $\mathrm{S} 235^{\mathrm{e})}$ & 26 & 235 & 284 & 1.10 \\
\hline S355 e) & 19 & 355 & 410 & 1.08 \\
\hline
\end{tabular}


Grade 300 g)

Grade 500 g)

320

500

523

1.02

a) provided by XLam NZ Ltd, b) provided by Nelson Pine Industries Ltd with grades according to AS/NZS 4357.0:2005 (2005) ${ }^{\text {c) }}$ EN336 (2016) with assumed normal distribution, ${ }^{\mathrm{d})}$ holds for $\rho \in[300,600] \mathrm{kg} / \mathrm{m}^{3}, d \epsilon[6,30] \mathrm{mm}, t / d \epsilon[2,10]$, ${ }^{\mathrm{e})}$ Karmazínová and Melcher (2012), ${ }^{\text {f) }}$ allowable range AS/NZS 4671:2001 (2001), g) tensile test data provided by Pacific Steel. 
Table 3. Material input properties, strength predictions and theoretical overstrength.

\begin{tabular}{|c|c|c|c|c|c|c|c|}
\hline & \multicolumn{5}{|c|}{ small-scale hold-downs } & \multicolumn{2}{|c|}{ large-scale hold-downs } \\
\hline & & nails & nails & dowels & dowels & dowels & dowels \\
\hline property & & CLT & LVL & CLT & LVL & CLT & CLT-LVL \\
\hline$\rho_{k}$ & {$\left[\mathrm{~kg} / \mathrm{m}^{3}\right]$} & 400 & 500 & 400 & 500 & 400 & $400-480$ \\
\hline$d$ & {$[\mathrm{~mm}]$} & 4 & 4 & 20 & 20 & 20 & 20 \\
\hline$t_{1}\left(L_{p}\right)$ & {$[\mathrm{mm}]$} & 90 & 90 & 55 & 56.5 & 85 & $83(40-43)$ \\
\hline$f_{h, k}$ & [MPa] & 30.4 & 39.4 & 22.6 & 34.7 & 22.6 & 28.2 \\
\hline$f_{y, n o m}$ & {$[\mathrm{MPa}]$} & 530 & 530 & 300 & 300 & 300 & 300 \\
\hline$M_{y}$ & {$[\mathrm{Nm}]$} & 5.7 & 5.7 & 400 & 400 & 400 & 400 \\
\hline$n$ & fasteners & 15 & 15 & 4 & 4 & 16 & 16 \\
\hline$F_{\text {rope }}$ & {$[\mathrm{kN}]$} & $25 \%$ a) & $25 \%$ a) & 0 b) & $0^{b)}$ & $2 \times 25 \% c)$ & $2 \times 25 \% c)$ \\
\hline$F_{u, k, p r e d}$ & {$[\mathrm{kN}]$} & 71 & 81 & 155 & 204 & 734 & 854 \\
\hline$F_{u, 0.05}$ & {$[\mathrm{kN}]$} & 71 & 81 & 160 & 209 & 750 & 879 \\
\hline$F_{u, 0.95}$ & {$[\mathrm{kN}]$} & 104 & 103 & 224 & 240 & 1106 & 1135 \\
\hline$\gamma_{0.95}$ & {$[-]$} & 1.46 & 1.27 & 1.40 & 1.15 & 1.48 & 1.29 \\
\hline$f_{h, k, c a l c}$ & {$[\mathrm{MPa}]$} & 30.4 & 39.4 & 24.4 & - & 24.0 & 28.2 \\
\hline
\end{tabular}




\begin{tabular}{llllllll}
$f_{h, 0.05, \text { exp }}$ & {$[\mathrm{MPa}]$} & - & - & 26.2 & - & 25.1 & 32.1 \\
$f_{\text {y,nom }}$ & {$[\mathrm{MPa}]$} & - & - & 300 & 300 & 300 & 300 \\
$f_{\text {, } 0.05}$ & {$[\mathrm{MPa}]$} & 530 & 530 & 320 & 320 & 320 & 320 \\
$\gamma_{\text {mod }}$ & {$[-]$} & 1.05 e) & 1.05 e) & 1.05 & $1.05^{\mathrm{a}}$ & 1.07 & 1.13 \\
$\gamma_{\text {Rd,th }}$ & {$[-]$} & $\mathbf{1 . 5 3}$ & $\mathbf{1 . 3 3}$ & $\mathbf{1 . 4 7}$ & $\mathbf{1 . 2 1}$ & $\mathbf{1 . 5 8}$ & $\mathbf{1 . 4 5}$ \\
$\gamma_{\text {err }}$ & {$[-]$} & $\mathrm{n} / \mathrm{a}$ & $\mathrm{n} / \mathrm{a}$ & $\mathrm{n} / \mathrm{a}$ & $\mathrm{n} / \mathrm{a}$ & 1.17 & 1.15 \\
\hline
\end{tabular}

a) rope effect nails: $F_{\text {rope }}=\min \left(F_{a x} / 4,0.25 F_{u, k}\right)$ b) all smooth dowels ${ }^{\text {c) }} 2$ dowels with threaded ends and nuts and washers in end row, ${ }^{\text {e) }}$ conservative assumption based on other results, $\mathrm{n} / \mathrm{a}=$ not applicable. 
Table 4. Experimental results and validation of $\gamma_{R d, t h}$.

\begin{tabular}{|c|c|c|c|c|c|c|}
\hline & $F_{\max , \exp }[\mathrm{kN}]$ & $F_{u, k, p r e d}[\mathrm{kN}]$ & $\mu[-]$ & $\gamma_{R d, \exp }[-]$ & $\gamma_{R d, t h}[-]$ & $\gamma_{R d, t h} \gamma_{\text {err }}[-]$ \\
\hline \multicolumn{7}{|c|}{ Nailed hold-downs in CLT - wide (Ottenhaus et al. 2016) } \\
\hline MON01 & 88.6 & 71 & 11.1 & 1.24 & 1.53 & $\mathrm{n} / \mathrm{a}$ \\
\hline MON02 & 96.4 & 71 & 8.4 & 1.35 & 1.53 & $\mathrm{n} / \mathrm{a}$ \\
\hline MON03 & 93.9 & 71 & 6.9 & 1.32 & 1.53 & $\mathrm{n} / \mathrm{a}$ \\
\hline ISO01 & 96.9 & 71 & 7.7 & 1.36 & 1.53 & $\mathrm{n} / \mathrm{a}$ \\
\hline ISO02 & 88.1 & 71 & 6.2 & 1.24 & 1.53 & $\mathrm{n} / \mathrm{a}$ \\
\hline ISO03 & 94.2 & 71 & 6.7 & 1.32 & 1.53 & $\mathrm{n} / \mathrm{a}$ \\
\hline CUREE04 & 86.1 & 71 & 6.7 & 1.21 & 1.53 & $\mathrm{n} / \mathrm{a}$ \\
\hline
\end{tabular}

Nailed hold-downs in LVL - dense (Ottenhaus et al. 2016)

\begin{tabular}{lcccccc}
\hline MON01 & 89.5 & 81 & 8.3 & 1.10 & 1.33 & n/a \\
MON02 & 97.1 & 81 & 10.5 & 1.19 & 1.33 & n/a \\
MON03 & 88.1 & 81 & 11.8 & 1.08 & 1.33 & n/a \\
& & & & & & \\
ISO02 & 101.4 & 81 & 10.8 & 1.25 & 1.33 & n/a \\
& & & & & & \\
ISO03 & 88.2 & 81 & 9.1 & 1.08 & 1.33 & $\mathrm{n} / \mathrm{a}$ \\
CUREE01 & 90.7 & 81 & 10.4 & 1.11 & 1.33 & $\mathrm{n} / \mathrm{a}$
\end{tabular}




$\begin{array}{lcccccc}\text { CUREE02 } & 102.8 & 81 & 8.7 & 1.26 & 1.33 & \mathrm{n} / \mathrm{a} \\ & & & & & & \\ \text { CUREE03 } & 99.5 & 81 & 6.8 & 1.22 & 1.33 & \mathrm{n} / \mathrm{a}\end{array}$

Small-scale dowelled hold-downs in CLT (Ottenhaus et al. 2017, 2018a)

\begin{tabular}{|c|c|c|c|c|c|c|}
\hline L1-M01 & 195 & 155.4 & 20.8 & 1.25 & 1.47 & $\mathrm{n} / \mathrm{a}$ \\
\hline L1-M03 & 194 & 155.4 & 7.5 & 1.25 & 1.47 & $\mathrm{n} / \mathrm{a}$ \\
\hline L1-M06 & 242 & 155.4 & 41.8 & 1.56 & 1.47 & $\mathrm{n} / \mathrm{a}$ \\
\hline L1-C01 & 193 & 155.4 & 10.4 & 1.24 & 1.47 & $\mathrm{n} / \mathrm{a}$ \\
\hline L1-C02 & 195 & 155.4 & 14.4 & 1.25 & 1.47 & $\mathrm{n} / \mathrm{a}$ \\
\hline L1-C04 & 171 & 155.4 & 7.9 & 1.10 & 1.47 & $\mathrm{n} / \mathrm{a}$ \\
\hline L1-C05 & 162 & 155.4 & 15.4 & 1.04 & 1.47 & $\mathrm{n} / \mathrm{a}$ \\
\hline L2-M01 & 224 & 155.4 & 24.4 & 1.44 & 1.47 & $\mathrm{n} / \mathrm{a}$ \\
\hline L2-M02 & 203 & 155.4 & 17.9 & 1.31 & 1.47 & $\mathrm{n} / \mathrm{a}$ \\
\hline L2-M04 & 194 & 155.4 & 24.5 & 1.25 & 1.47 & $\mathrm{n} / \mathrm{a}$ \\
\hline L3-M02 & 168 & 155.4 & 9.0 & 1.08 & 1.47 & $\mathrm{n} / \mathrm{a}$ \\
\hline L3-M03 & 164 & 155.4 & 9.5 & 1.06 & 1.47 & $\mathrm{n} / \mathrm{a}$ \\
\hline L3-M04 & 187 & 155.4 & 9.3 & 1.20 & 1.47 & $\mathrm{n} / \mathrm{a}$ \\
\hline L3-M05 & 186 & 155.4 & 6.5 & 1.20 & 1.47 & $\mathrm{n} / \mathrm{a}$ \\
\hline
\end{tabular}


Small-scale dowelled hold-downs in LVL (Ottenhaus et al. 2017, 2018a)

\begin{tabular}{lcccccc}
\hline L1-M04 & 210 & 204.0 & 10.0 & 1.03 & 1.21 & $\mathrm{n} / \mathrm{a}$ \\
L1-C01 & 205 & 204.0 & 9.7 & 1.01 & 1.21 & $\mathrm{n} / \mathrm{a}$ \\
L1-C02 & 209 & 204.0 & 9.7 & 1.02 & 1.21 & $\mathrm{n} / \mathrm{a}$ \\
L1-C03 & 204 & 204.0 & 15.5 & 1.00 & 1.21 & $\mathrm{n} / \mathrm{a}$ \\
L2-M02 & 215 & 204.0 & 8.1 & 1.05 & 1.21 & $\mathrm{n} / \mathrm{a}$ \\
L2-M04 & 216 & & & & & \\
L2-M05 & 229 & 204.0 & 7.6 & 1.06 & 1.21 & $\mathrm{n} / \mathrm{a}$ \\
\hline
\end{tabular}

Large-scale dowelled hold-downs (Ottenhaus et al. 2018c)

\begin{tabular}{lcccccc}
\hline L2-01M & 1310.9 & 854 & 6.3 & $\mathbf{1 . 5 4}$ & 1.45 & 1.67 \\
L2-02C & 1291.8 & 854 & 6.5 & $\mathbf{1 . 5 1}$ & 1.45 & 1.67 \\
L2-03C & 1238.8 & 854 & 6.0 & 1.45 & 1.45 & 1.67 \\
& & & & & & \\
L2-04C & 1268 & 854 & 6.3 & $\mathbf{1 . 4 9}$ & 1.45 & 1.67 \\
& & & & & & \\
L3-01M & 1285.6 & 747 & 7.1 & $\mathbf{1 . 7 2}$ & 1.58 & 1.85 \\
& & & & & & \\
L3-04C & 1177.3 & 747 & 6.0 & 1.58 & 1.58 & 1.85 \\
\hline
\end{tabular}


Table 5. Validation of $\gamma_{R d, t h}$ with data from Jorissen (1998).

\begin{tabular}{|c|c|c|c|c|c|c|c|}
\hline $\begin{array}{l}\text { series } \\
\text { name }\end{array}$ & $\begin{array}{l}t_{1} \\
{[\mathrm{~mm}]}\end{array}$ & $\begin{array}{l}t_{2} \\
{[\mathrm{~mm}]}\end{array}$ & $\begin{array}{l}F_{\text {max }, \exp } \\
{[\mathrm{kN}]}\end{array}$ & $\begin{array}{l}F_{u, k, p r e d} \\
{[\mathrm{kN}]}\end{array}$ & $\begin{array}{l}\mu \\
{[-]}\end{array}$ & $\begin{array}{l}\gamma_{R d, \exp } \\
{[-]}\end{array}$ & $\begin{array}{c}\gamma_{R d, t h} \\
{[-]}\end{array}$ \\
\hline D31107 & 24 & 48 & 14.22 & 10.43 & 6.00 & 1.36 & 1.42 \\
\hline D33137 & 24 & 48 & 35.29 & 31.29 & 4.69 & 1.13 & 1.42 \\
\hline D33157 & 24 & 48 & 34.26 & 31.29 & 5.59 & 1.09 & 1.42 \\
\hline D35137 & 24 & 48 & 60.82 & 52.16 & 4.38 & 1.17 & 1.42 \\
\hline D35157 & 24 & 48 & 60.44 & 52.16 & 4.45 & 1.16 & 1.42 \\
\hline D41107 & 36 & 48 & 12.10 & 11.87 & 6.67 & 1.02 & 1.44 \\
\hline D45157 & 36 & 48 & 62.10 & 59.36 & 5.22 & 1.05 & 1.44 \\
\hline D61107 & 48 & 48 & 16.06 & 13.83 & 4.00 & 1.16 & 1.45 \\
\hline D65157 & 48 & 48 & 72.34 & 69.14 & 4.12 & 1.05 & 1.45 \\
\hline D81107 & 59 & 72 & 24.03 & 15.46 & 5.00 & 1.55 & 1.41 \\
\hline D83137 & 59 & 72 & 49.88 & 46.39 & 4.55 & 1.08 & 1.41 \\
\hline D83157 & 59 & 72 & 50.49 & 46.39 & 4.17 & 1.09 & 1.41 \\
\hline D89157 & 59 & 72 & 186.32 & 139.17 & 4.56 & 1.34 & 1.41 \\
\hline
\end{tabular}


Table 6. Validation of $\gamma_{R d, t h}$ with data from Dorn et al. (2013).

\begin{tabular}{lllllll}
\hline $\begin{array}{l}\text { series } \\
\text { name }\end{array}$ & $t$ & $F_{\max , \exp }$ & $F_{u, k, \text { pred }}$ & $\mu$ & $\gamma_{\text {Rd,exp }}$ & $\gamma_{R d, \text { th }}$ \\
\hline 1 & 45 & {$[\mathrm{kN}]$} & {$[\mathrm{kN}]$} & {$[-]$} & {$[-]$} & {$[-]$} \\
\hline 2 & 45 & 27.50 & 19.48 & $\mathrm{D}$ & 1.41 & 1.51 \\
$08 \mathrm{a}$ & 45 & 30.00 & 19.48 & $\mathbf{D}$ & $\mathbf{1 . 5 4}$ & 1.51 \\
10 & 45 & 24.00 & 19.48 & $\mathrm{D}$ & 1.23 & 1.51 \\
$16 \mathrm{a}$ & 45 & 23.00 & 19.48 & HD & 1.18 & 1.51 \\
$16 \mathrm{c}$ & 45 & 20.00 & 19.48 & HD & 1.03 & 1.51 \\
\hline
\end{tabular}




\section{Figure Captions}

Fig. 1 Theoretical overstrength components (modified from Jorissen and Fragiacomo (2011)). Figure by Ottenhaus (2019).

Fig. 2 Experimentally derived overstrength. Figure by Ottenhaus (2019a).

Fig. 3 European Yield Model for steel-to-timber connection with a) thick steel side plate and b) internal steel plate.

Fig. 4. a) Overstrength components. Figure by Ottenhaus (2019b), b) overstrength from variability in material properties and sources. Figure by Ottenhaus (2019c), c) overstrength from conservatism in analytical models and contributing sources. Figure by Ottenhaus (2019d).

Fig. 5 Model overstrength $\gamma_{\text {mod,Meff }}$ from using the effective bending moment instead of plastic bending moment.

Fig. 6 Load displacement curves, predicted strength, $F_{u, k, p r e d}$, upper bound overstrength $\gamma_{R d, t h} F_{u, k \text { pred }}$, for a) nailed connections in CLT, b) nailed connections in LVL, c) dowelled small-scale connections in CLT, d) dowelled small-scale connections in LVL. 


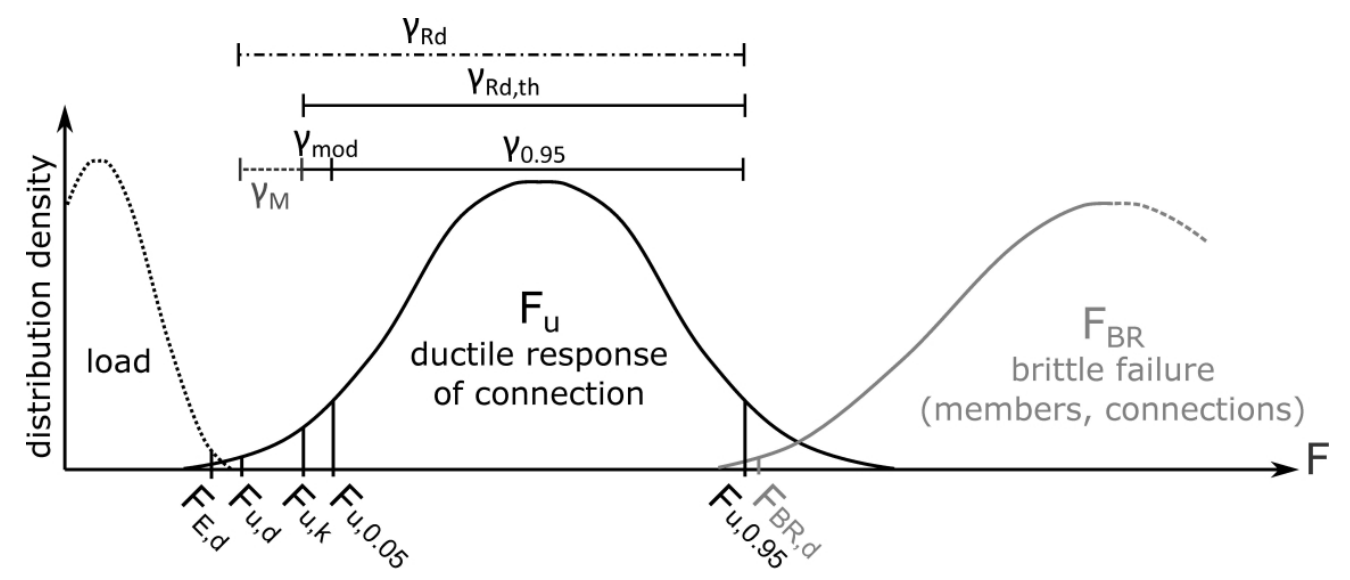

Theoretical overstrength components (modified from Jorissen and Fragiacomo (2011)). Figure by Ottenhaus (2019).

$232 \times 98 \mathrm{~mm}(600 \times 600 \mathrm{DPI})$ 


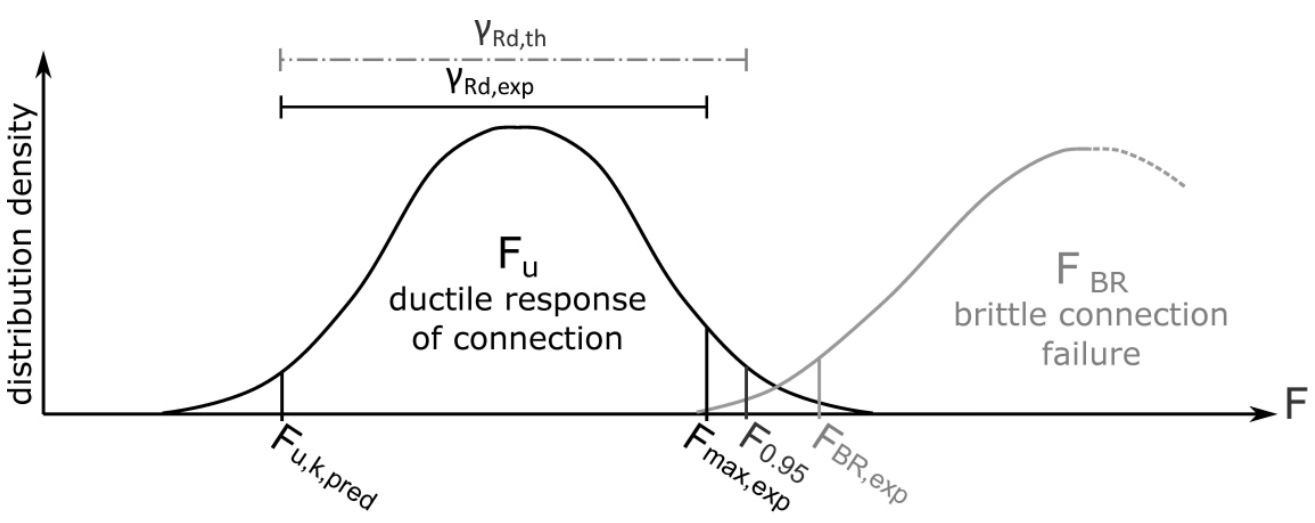

Experimentally derived overstrength. Figure by Ottenhaus (2019a).

$232 \times 89 \mathrm{~mm}(600 \times 600 \mathrm{DPI})$ 

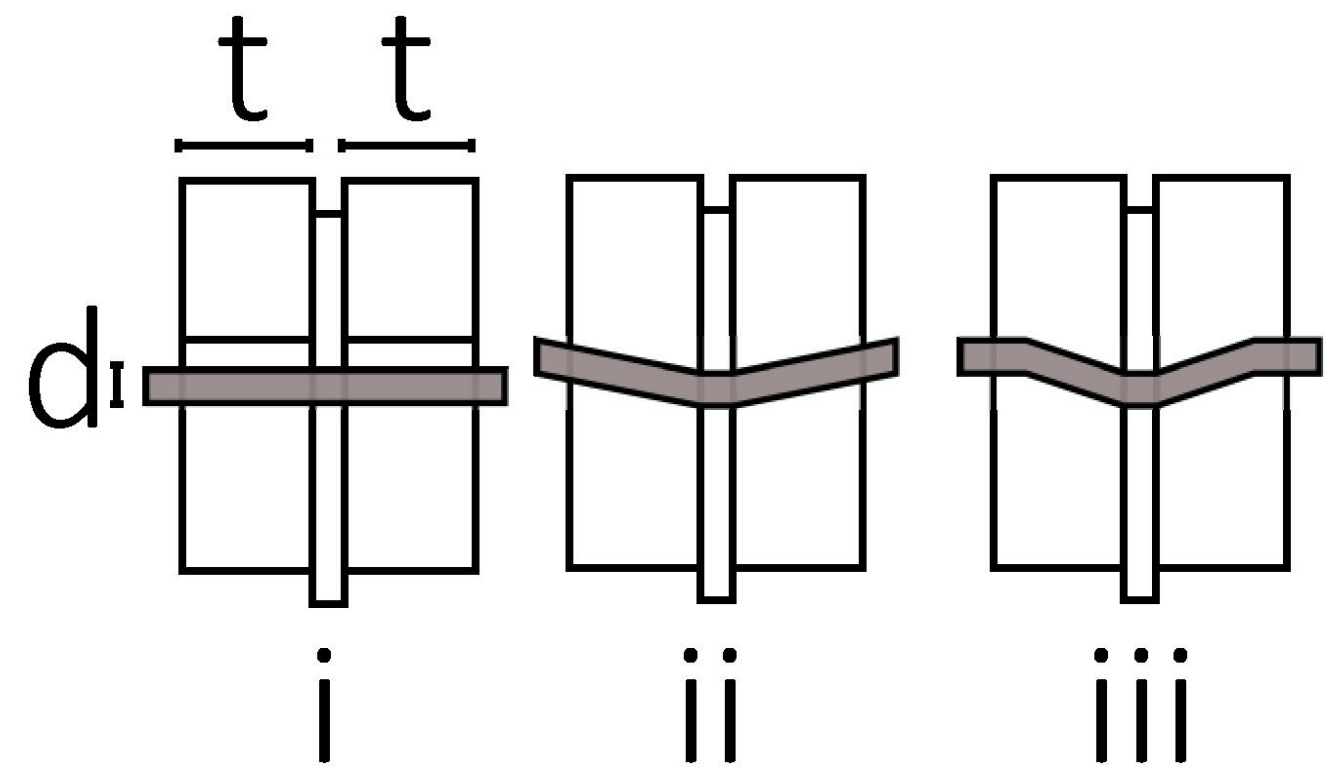

European Yield Model for steel-to-timber connection with a) thick steel side plate and b) internal steel plate. $56 \times 32 \mathrm{~mm}(600 \times 600 \mathrm{DPI})$ 

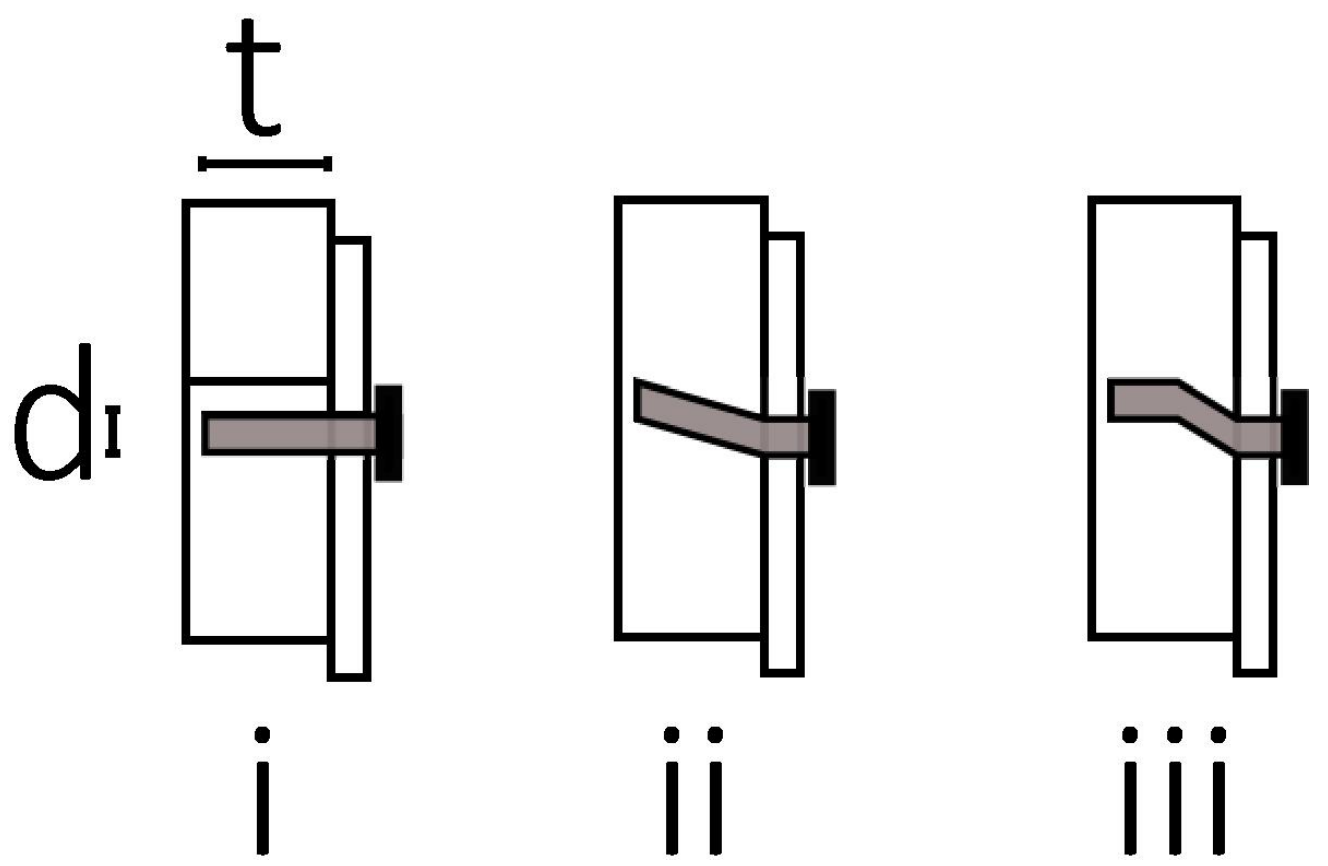

European Yield Model for steel-to-timber connection with a) thick steel side plate and b) internal steel plate. $50 \times 32 \mathrm{~mm}(600 \times 600$ DPI $)$ 


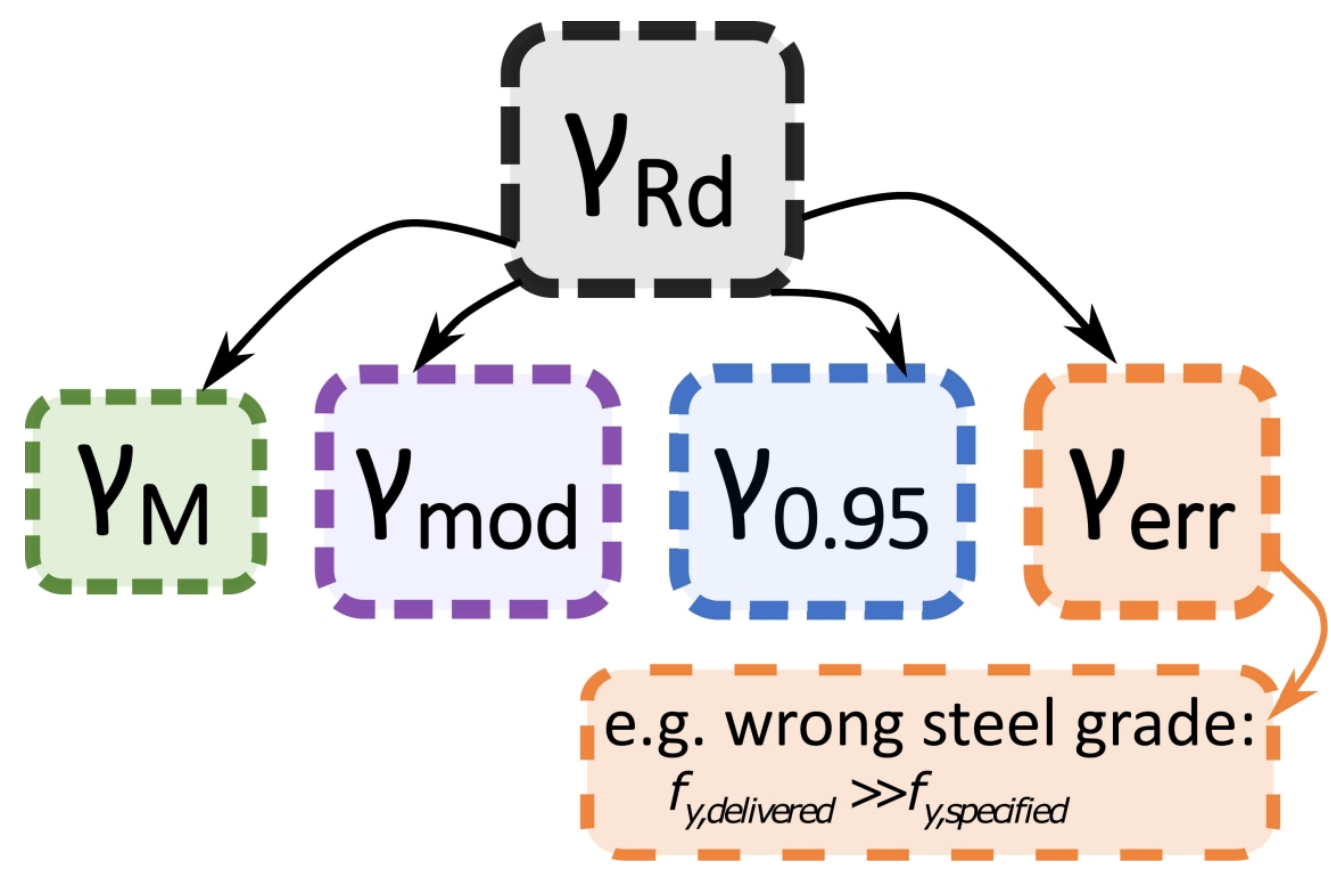

a) Overstrength components. Figure by Ottenhaus (2019b), b) overstrength from variability in material properties and sources. Figure by Ottenhaus (2019c), c) overstrength from conservatism in analytical models and contributing sources. Figure by Ottenhaus (2019d).

$229 \times 148 \mathrm{~mm}(600 \times 600 \mathrm{DPI})$ 
a) Overstrength components. Figure by Ottenhaus (2019b), b) overstrength from variability in material properties and sources. Figure by Ottenhaus (2019c), c) overstrength from conservatism in analytical models and contributing sources. Figure by Ottenhaus (2019d).

$307 \times 214 \mathrm{~mm}(600 \times 600 \mathrm{DPI})$ 
a) Overstrength components. Figure by Ottenhaus (2019b), b) overstrength from variability in material properties and sources. Figure by Ottenhaus (2019c), c) overstrength from conservatism in analytical models and contributing sources. Figure by Ottenhaus (2019d).

$298 \times 215 \mathrm{~mm}(600 \times 600 \mathrm{DPI})$ 


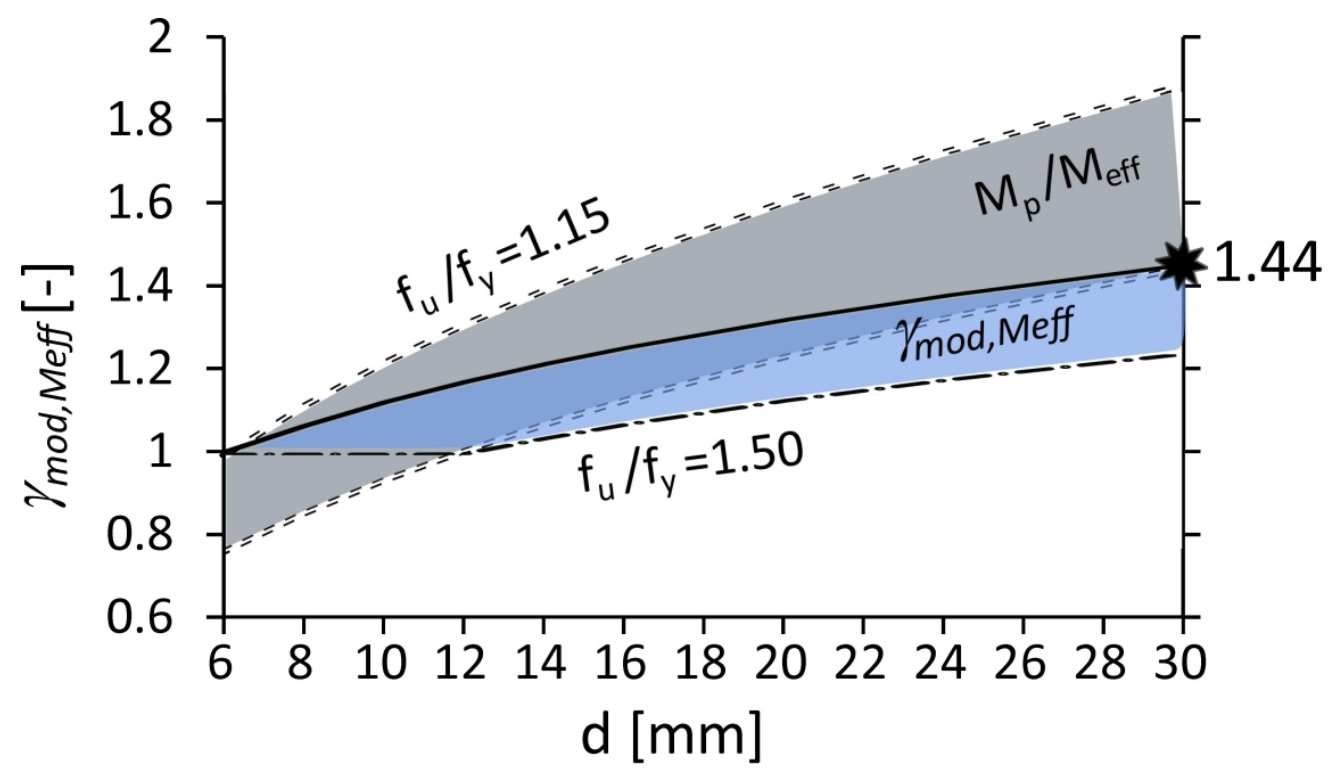

Model overstrength ymod,Meff from using the effective bending moment instead of plastic bending moment.

$174 \times 100 \mathrm{~mm}(600 \times 600 \mathrm{DPI})$ 


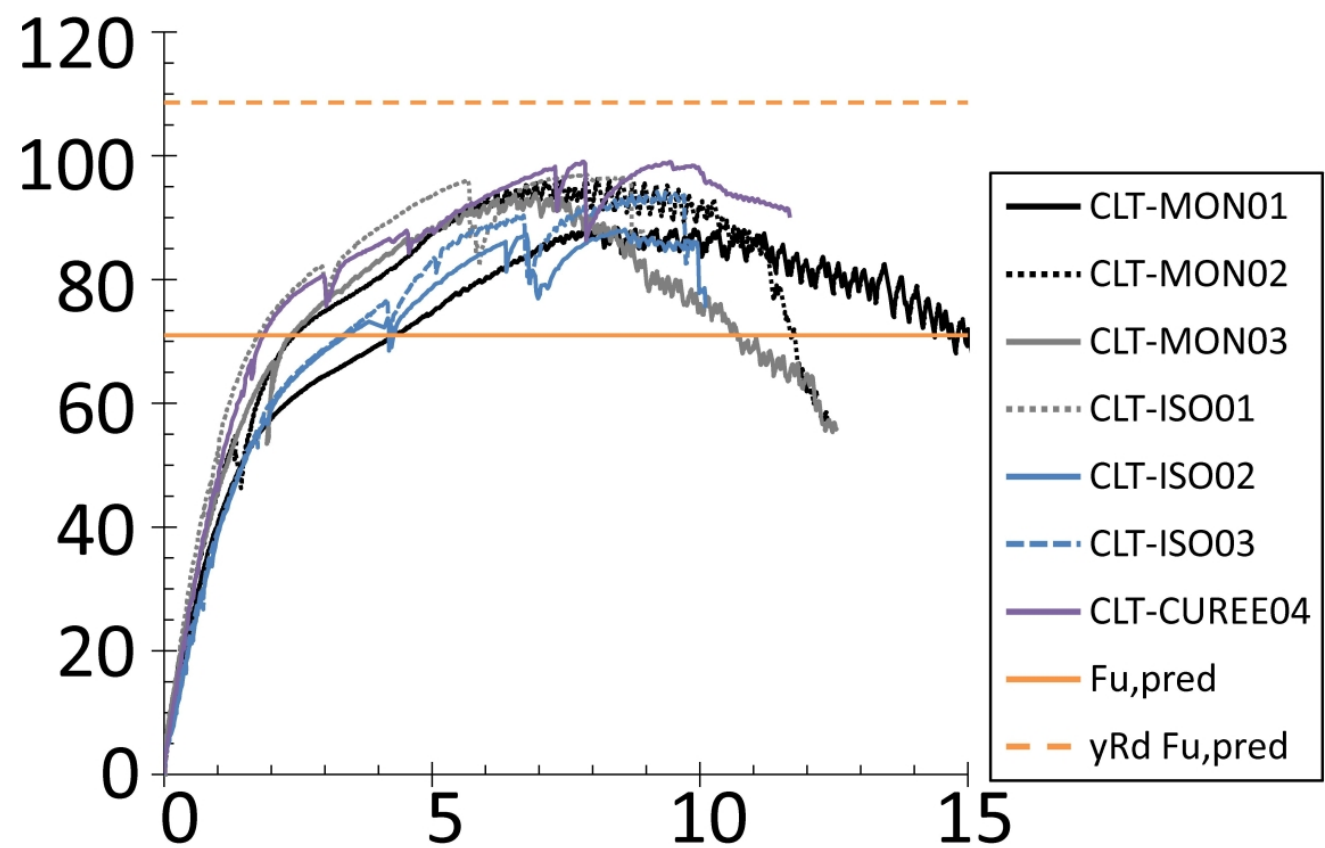

Load displacement curves, predicted strength, Fu,k,pred, upper bound overstrength $y$ Rd,th Fu,k,pred, for a) nailed connections in CLT, b) nailed connections in LVL, c) dowelled small-scale connections in CLT, d) dowelled small-scale connections in LVL.

$197 \times 130 \mathrm{~mm}(600 \times 600 \mathrm{DPI})$ 


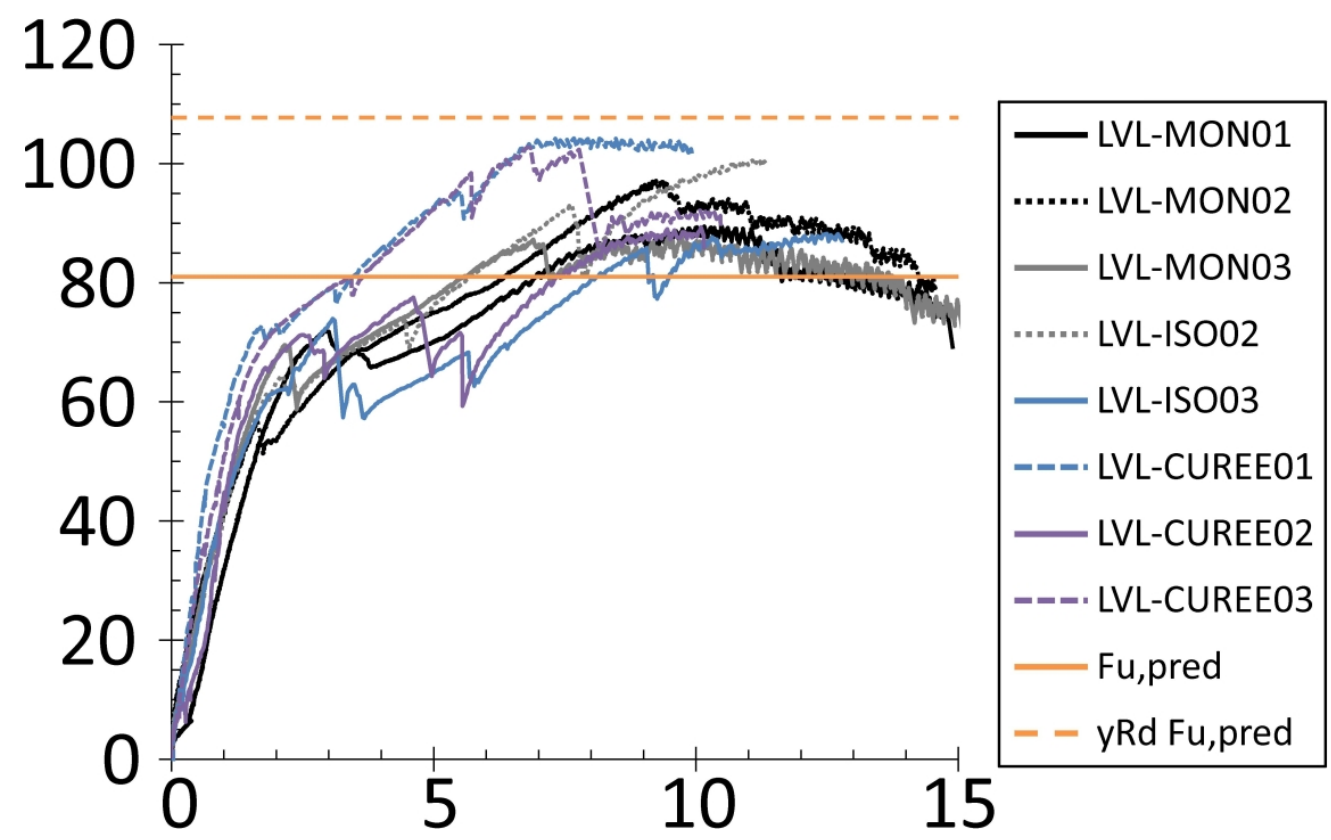

Load displacement curves, predicted strength, Fu,k,pred, upper bound overstrength $y$ Rd,th Fu, $k$,pred, for a) nailed connections in CLT, b) nailed connections in LVL, c) dowelled small-scale connections in CLT, d) dowelled small-scale connections in LVL. 


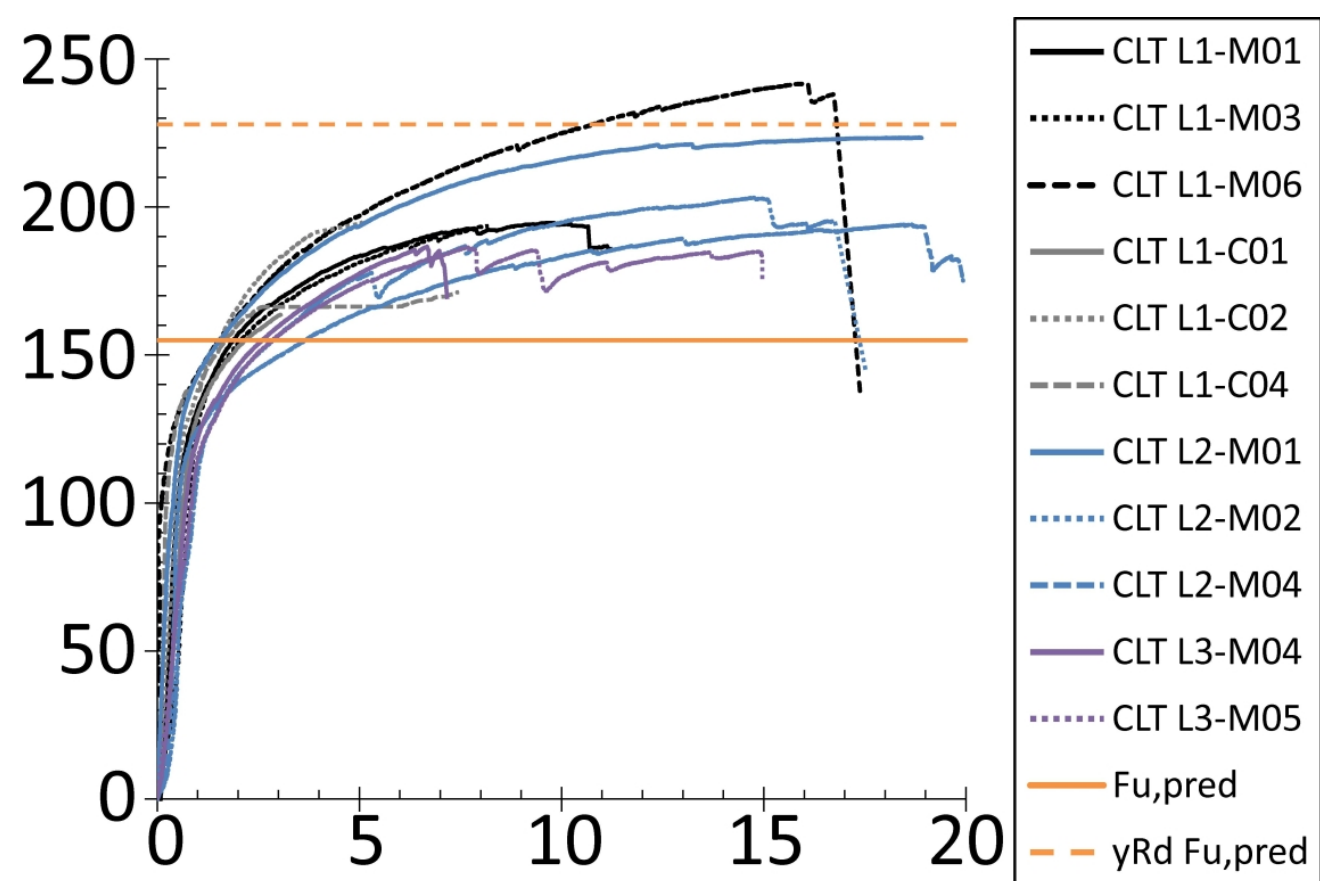

Load displacement curves, predicted strength, Fu,k,pred, upper bound overstrength $y$ Rd, th Fu,k, pred, for a) nailed connections in CLT, b) nailed connections in LVL, c) dowelled small-scale connections in CLT, d) dowelled small-scale connections in LVL.

$199 \times 133 \mathrm{~mm}(600 \times 600 \mathrm{DPI})$ 


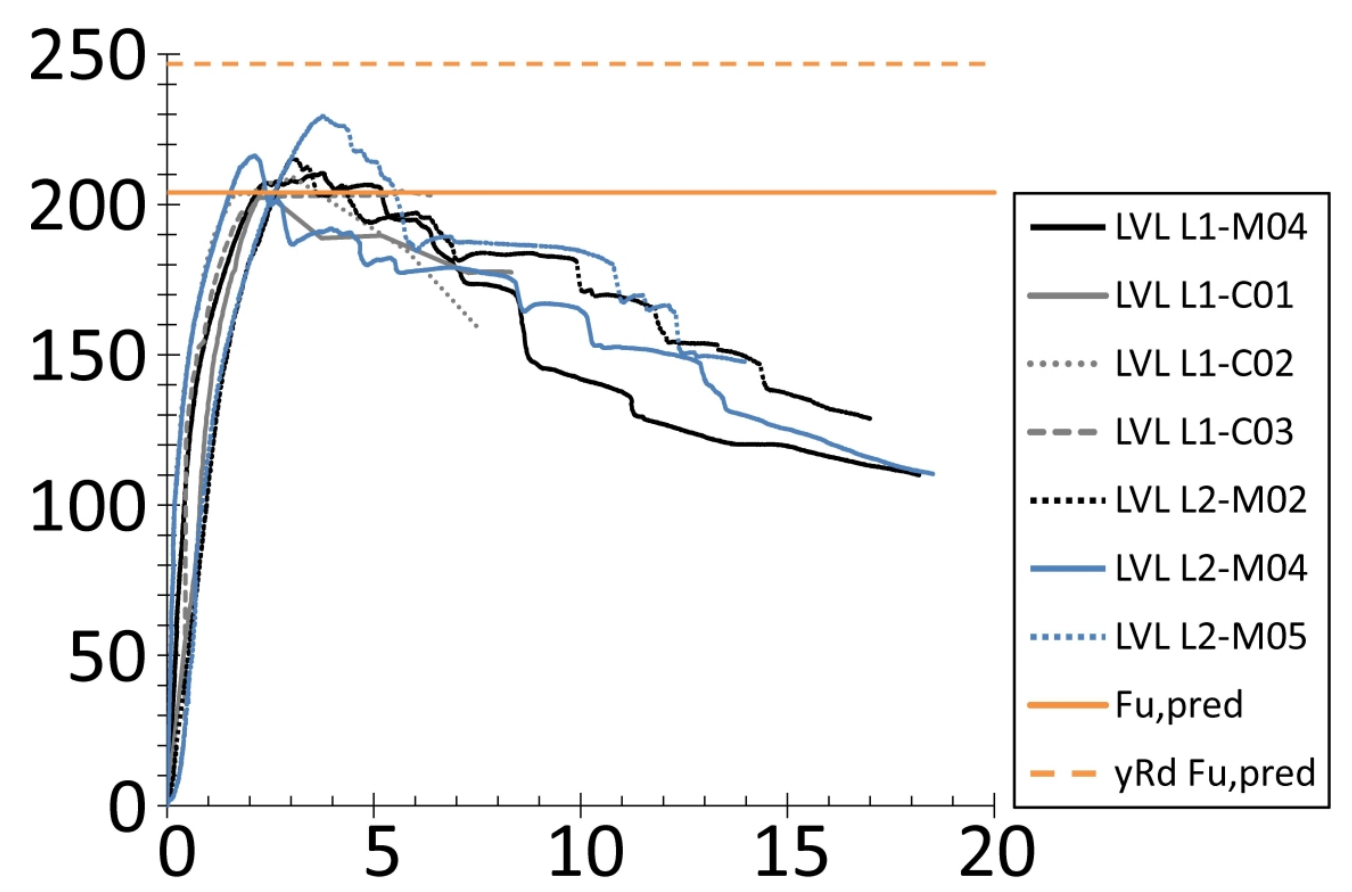

Load displacement curves, predicted strength, Fu,k,pred, upper bound overstrength $\gamma R d$,th Fu,k,pred, for a) nailed connections in CLT, b) nailed connections in LVL, c) dowelled small-scale connections in CLT, d) dowelled small-scale connections in LVL.

$$
195 \times 130 \mathrm{~mm}(600 \times 600 \text { DPI })
$$

